\title{
Dynamics from a predator-prey-quarry-resource-scavenger model
}

\author{
Joanneke E. Jansen ${ }^{1}$ - Robert A. Van Gorder ${ }^{1}$ (D)
}

Received: 23 June 2017 / Accepted: 21 August 2017 / Published online: 14 September 2017

(C) The Author(s) 2017. This article is an open access publication

\begin{abstract}
Allochthonous resources can be found in many foodwebs and can influence both the structure and stability of an ecosystem. In order to better understand the role of how allochthonous resources are transferred as quarry from one predator-prey system to another, we propose a predator-prey-quarry-resource-scavenger (PPQRS) model, which is an extension of an existing model for quarryresource-scavenger (a predator-prey-subsidy (PPS) model). Instead of taking the allochthonous resource input rate as a constant, as has been done in previous theoretical work, we explicitly incorporated the underlying predator-prey relation responsible for the input of quarry. The most profound differences between PPS and PPQRS system are found when the predator-prey system has limit cycles, resulting in a periodic rather than constant influx of quarry (the allochthonous resource) into the scavenger-resource interactions. This suggests that the way in which allochthonous resources are input into a predator-prey system can have a strong influence over the population dynamics. In order to understand the role of seasonality, we incorporated non-autonomous terms and showed that these terms can either stabilize or destabilize the dynamics, depending on the parameter regime. We also considered the influence of spatial motion (via diffusion) by constructing a continuum partial differential equation (PDE) model over space. We determine when
\end{abstract}

The PDF is for review purposes only. The Latex file is uploaded as supplementary material.

Robert A. Van Gorder

Robert.VanGorder@maths.ox.ac.uk

1 Mathematical Institute, University of Oxford, Andrew Wiles Building, Radcliffe Observatory Quarter, Woodstock Road, Oxford OX2 6GG, UK such spatial dynamics essentially give the same information as the ordinary differential equation (ODE) system, versus other cases where there are strong spatial differences (such as spatial pattern formation) in the populations. In situations where increasing the carrying capacity in the ODE model drives the amplitude of the oscillations up, we found that a large carrying capacity in the PDE model results in a very small variation in average population size, showing that spatial diffusion is stabilizing for the PPQRS model.

Keywords Predator-prey dynamics · Allochthonous resource subsidy · Population dynamics · Non-equilibrium dynamics $\cdot$ Chaotic oscillations

\section{Introduction}

The classic predator-prey models of Lotka (1925) and Volterra (1926) are cornerstones of mathematical ecology. Since they were first proposed in the early 1900s, they have been studied extensively. Their models have been improved, leading to more realistic models such as, amongst others (see Berryman (1992) for a review), the equations of Solomon (1949), Holling (1959, 1965), Real (1977) and Leslie (1948). Models with two prey and one predator, known as apparent competition models, are also well studied theoretically (Holt 1977; Vandermeer 2006). Instead of the addition of an extra prey, a two species model can also be extended by the addition of an allochthonous resource. The use of allochthonous resources from nearby communities is important in many ecosystems (Huxel et al. 2002). Examples include Californian coyotes that base a large part of their diet on aquatic food (Rose and Polis 1998), trouts that feed on mosquito's (Berg and Hellenthal 1992), and river-dwelling organisms that receive organic carbon from 
upstream sources (Vannote et al. 1980; Huxel et al. 2002). To understand the influence of allochthonous resources on ecosystem dynamics, several predator-prey-subsidy (PPS) models have been studied (Huxel and McCann 1998; Huxel et al. 2002; Pang and Wang 2004; Nevai and Van Gorder 2012). In those models, the subsidy is treated as an external food source that enters the ecosystem at a rate that is independent of other species dynamics. In reality, the input rate of the subsidy will depend on species dynamics of a nearby ecosystem.

In this paper, we will consider a model that consists of two independent predator-prey relations, where part of the prey of the first system is introduced as an allochthonous resource into the second system. We will call our five-dimensional model a predator-prey-quarryresource-scavenger (PPQRS) system. Here, part of the prey is eaten by the predator and another part of it, in the form of quarry, by the scavenger, whose primary food source is the resource. Note that in PPS systems, the PP ecosystem is subsidized, whereas in our PPQRS model, the PP (sub-)system subsidizes another PP subsystem in which the scavengers (S) are the predators and the resources $(\mathrm{R})$ the prey.

Our motivating example throughout this paper will be the Arctic ecosystem. We will extend the model used in Nevai and Van Gorder (2012). They studied an autonomous predator-prey model involving Arctic foxes (predator), lemmings (prey) and seal carcasses (subsidy). The primary prey of Arctic foxes (Alopex lagopus) are lemmings (Cricetidae family). During the winter months, when the lemming population is low, foxes use the carcasses of seals (Phocidae family) left by polar bears (Usrsus martimus) as a subsidary food source (Roth 2002; 2003). So, in our PPQRS model, the polar bears will act as the predator, the seals as the prey, the seal carcasses as the quarry, the lemmings as the resource and the foxes, finally, as the scavenger.

We compare our results with the ones as found by Nevai and Van Gorder for their autonomous PPS model (Nevai and Van Gorder 2012) and with the ones as found by Levy et al. (2016) for their PPS model with oscillating subsidy input rate. We will also discuss the difference between the PPQRS model and apparent competition models, which we will find to look very similar to our system. The most profound differences between PPS and PPQRS system are found when the predator-prey system has limit cycles, resulting in a periodic rather than constant influx of quarry (the allochthonous resource) into the scavenger-resource interactions. This suggests that the way in which allochthonous resources are input into a predator-prey system can have a strong influence over the population dynamics.

We will start with an introduction of the ordinary differential equation (ODE) model in "The autonomous PPQRS model". We examine the steady states and their stability in "Steady and non-equilibrium dynamics", and then consider numerical simulations for non-equilibrium dynamics. In "Non-constant quarry input rate", we focus on dynamics equivalent to their being a non-constant subsidy input rate and obtain non-equilibrium dynamics and chaos. In "PPQRS model with seasonality", we consider a nonautonomous form of the model in order to study the influence of seasonality on the PPQRS dynamics. In "Exten- sion to a spatial PDE model", we extend the ODE model to a partial differential equation (PDE) model, in order to better study spatial separation of the quarry and the resource. In "Discussion and biological implications", we discuss the mathematical results and their biological implications. Finally, in "Conclusions", we give brief conclusions.

\section{The autonomous PPQRS model}

The PPQRS model we will study in this paper is given by the following five-dimensional system of autonomous ODEs:

$$
\begin{aligned}
\frac{d u}{d t} & =\rho u\left(1-\frac{u}{c}\right)-\phi\left(\frac{u v}{u+g}\right), \\
\frac{d v}{d t} & =\left(\frac{\zeta \phi u}{u+g}\right) v-\beta v, \\
\frac{d s}{d t} & =\mu \phi\left(\frac{u v}{u+g}\right)-\gamma s-\psi\left(\frac{s y}{x+s+h}\right), \\
\frac{d x}{d t} & =r x\left(1-\frac{x}{k}\right)-\theta\left(\frac{x y}{x+s+h}\right), \\
\frac{d y}{d t} & =\left(\frac{\epsilon \theta x+\eta \psi s}{x+s+h}\right) y-\delta y .
\end{aligned}
$$

Here, $u(t), v(t), x(t)$ and $y(t)$ are the population sizes of the seals (prey), polar bears (predator), lemmings (resource) and foxes (scavenger), respectively, and $s(t)$ is the amount of seal carrion (quarry) available for the foxes. We assume that $u, v, s, x, y \geq 0$ and $t \geq 0$.

The constants $c$ and $k$ are the carrying capacities of the prey and the resource and $\rho$ and $r$ are their respective growth rates. The parameters $\phi$ and $\theta$ are the maximum rates at which the predators and scavengers consume their prey. The mortality rate of the predator is denoted by $\beta$ and of the scavenger by $\delta$. The parameters $h$ and $g$ are half-saturation constants and $\gamma$ is the decay rate of the quarry. Finally, $\zeta, \epsilon$, $\eta$ and $\mu$ are conversion factors, which we assume to be positive and smaller than unity. We also assume that $\zeta \phi>\beta$ and either $\epsilon \theta>\delta$ or $\eta \psi>\delta$, so that $\frac{d v}{d t}>0$ and $\frac{d y}{d t}>0$ for sufficiently large $u$ and $y$, respectively. We assume that part of the killed prey is turned into quarry. Our model does not allow prey that die for other reasons to become available to the scavengers. This would require a separate death rate in the equation for $v$, and while this could be included, it is not standard for such models. 
The inflow of quarry comes from predation of the predators $u$ on the prey $v$ via the first term in Eq. 2.3. This term is what couples the predator-prey system to the resourcescavenger-quarry system.

The model (2.1)-(2.5) is an extended, modified version of the PPS model suggested in Nevai and Van Gorder (2012), which is given by Eqs. 2.4 and 2.5 with Eq. 2.3 replaced by

$$
\frac{d s}{d t}=i-\gamma s-\psi\left(\frac{s y}{x+s+h}\right) .
$$

Observe that this PPS model is identical to Eqs. 2.3-2.5, except for the constant input rate $i$, which we replaced by the term $\mu \phi\left(\frac{u v}{u+g}\right)$ in Eqs. 2.3-2.5. The PPS model is in its turn an extension of the Rosenzweig-MacArthur predatorprey model (Rosenzweig and MacArthur 1963; Gasull et al. 1997). The Rosenzweig-MacArthur model is one of the many predator-prey models that have been studied and proposed (Murray 2002, p. 88). We have chosen this Gausetype model (Gause et al. 1936), because it is more realistic than the classic predator-prey models by Lotka (1925) and Volterra (1926), but is still very simple. The multi-species functional response in Eqs. 2.1-2.5 is the same as was used in Nevai and Van Gorder (2012) and is a natural generalization of the Holling type II response to two distinct resource inputs for the scavenger.

We note that our system has similarities with an apparent competition model (two resources and one consumer), such as studied for example in Abrams et al. (1998). The difference is that in our case, the amount of quarry does not grow logistically, but depends on the separate predatorprey system. The quarry is also assumed to be decaying in time. We will discuss the consequences of these differences in "Comparison with apparent competition models". There are apparent similarities to nutrient cycling (DeAngelis 1980; Vitousek 1982) or nutrient recycling (DeAngelis et al. 1989), in that some of the remaining prey biomass can be transferred to the resource-scavenger-quarry system, although the dynamics are a bit different.

Throughout this paper, we will assume $\psi=5, \theta=5$, $\phi=5, h=1, \gamma=1, g=1, \beta=0.1, \zeta=0.1, \epsilon=0.1$, $\eta=0.1$ and $\delta=0.1$. These standard values are chosen because they are similar to the values as used in Nevai and Van Gorder (2012) and Levy et al. (2016), so we can make easy comparisons with their PPS models. When applied to a real biological system, either an Arctic system as considered throughout this paper, or any other system where allochthonous resources are important, their values should be adjusted accordingly. We did not assign standard values to $\rho, r, \mu, c$ and $k$, as they are useful parameters to vary in order to sample the variety of dynamics possible.

\section{Steady and non-equilibrium dynamics}

In Appendix A, we consider the feasibility, local and global stability of the equilibrium states of our autonomous model, given by Eqs. 2.1-2.5, where we define an equilibrium state as a solution of our model that is constant in time. In Table 1, we give a summary of our results. As stated in Appendix A, when $\underset{\sim}{c}<c<\tilde{c}$, the prey and predators are in a stable equilibrium and our input rate $i=\mu \rho \mathcal{\sim}\left(1-\frac{\mathcal{c}}{\tilde{c}}\right)$ is constant. We note that in this case, our model gives equivalent dynamics to those in Nevai and Van Gorder (2012).

In Fig. 1, we plot the bifurcation diagrams of the full dynamics of the system, including non-equilibrium dynamics (which may include limit cycles, quasi-periodic orbits and chaos). We took different values of $\mu$ and $\rho$. Above, we saw that the resource-free equilibrium $\left(u^{*}, v^{*}, \underline{l}, 0, \frac{\eta}{\delta}\left(i-\gamma s^{*}\right)\right)$ is only stable when both $c<\tilde{c}$ and $i>i^{*}=\left(\gamma+\frac{r \psi}{\theta}\right) \underline{l}$. For $\underset{\sim}{c}<c<\tilde{c}$, the constant input rate is given by $i=\mu \rho \mathcal{\sim}\left(1-\frac{c}{c}\right)$. Because $i$ is dependent on $c$, we see that the resource-free equilibrium will never be stable if $\mu \rho \underset{\sim}{c}\left(1-\frac{c}{\tilde{\tilde{c}}}\right)<\tilde{c}$. Indeed, we see in Fig. 1a-c that the resource-free equilibrium is never stable. We will now investigate what happens when we let $c>\tilde{c}$, so that the prey and predators will be in limit cycle and the input rate $i$ is not constant any more.

\section{Non-constant quarry input rate}

We now turn our attention to the case where the predatorprey system is in a limit cycle (as the dynamics are planar, this is the most complicated example of non-equilibrium dynamics possible). We shall attempt to understand how this will modify the resource-scavenger system for parameter values which would normally indicate steady states or limit cycles.

We consider three cases: (i) $\underset{\sim}{k}<k<\tilde{k}$ (so that the scavenger-resource system is in positive equilibrium if no quarry, (ii) $\tilde{k}>k$ (so that we have both a limit cycle from the predator-prey dynamics and one from the scavengerresource dynamics with no quarry), (iii) and finally $\tilde{k}>k$ with $\mu=0.9$ and $c=5$ (which should give even more extreme oscillations). To motivate the third case, it is known that the amplitude of the limit cycle of the predator-prey system grows for increasing $\tilde{c}<c$ (Hofbauer and Sigmund 1998). As the input rate of quarry is dependent on the predator-prey system, the amplitude of the input rate will also grow when $c$ grows. Further, by increasing the conversion factor $\mu$, we can increase the amplitude of the input rate of quarry even further.

In case (i), regular periodic dynamics are observed, due to the periodic forcing from the predator-prey system. In 
Table 1 Summary of steady-state properties of the autonomous PPQRS system; for derivations of the results, see Appendix A

\begin{tabular}{|c|c|c|c|c|c|c|}
\hline$u$ & $v$ & $s$ & $x$ & $y$ & Feasible & Stable \\
\hline 0 & 0 & 0 & 0 & 0 & Always & Never \\
\hline 0 & 0 & 0 & $k$ & 0 & Always & Never \\
\hline 0 & 0 & 0 & $x^{*}$ & $y^{*}$ & $k>\underset{\sim}{k}$ & Never \\
\hline$c$ & 0 & 0 & 0 & 0 & Always & Never \\
\hline$c$ & 0 & 0 & $k$ & 0 & Always & $c<\underset{\sim}{c}$ and $k<\underset{\sim}{k}$ \\
\hline$c$ & 0 & 0 & $x^{*}$ & $y^{*}$ & $k>\underset{\sim}{k}$ & $c<\underset{\sim}{c}$ and $k<\tilde{k}$ \\
\hline$u^{*}$ & $v^{*}$ & $i / \gamma$ & 0 & 0 & $c>\underset{\sim}{c}$ & Never \\
\hline$u^{*}$ & $v^{*}$ & $i / \gamma$ & $k$ & 0 & $c>\underset{\sim}{c}$ & $c<\tilde{c}, k<\underset{\sim}{k}$ and $i<i_{*}(k)$ \\
\hline$u^{*}$ & $v^{*}$ & $\underline{l}$ & 0 & $\eta / \delta(i-\gamma \underline{l})$ & $c>\underset{\sim}{c}$ and $i>\gamma \underline{l}$ & $c<\tilde{c}$ and $i>i^{*}$ \\
\hline$u^{*}$ & $v^{*}$ & $s^{*}$ & $x^{*}$ & $y^{*}$ & $\begin{array}{l}c>\underset{\sim}{c}, k<\underset{\sim}{k} \text { and } i_{*}(k)<i<i^{*} \\
c>\underset{\sim}{k}<<<\tilde{k} \text { and } i<i^{*} \\
c>\underset{\sim}{c}, k>\tilde{k} \text { and } i<i^{*}\end{array}$ & $\begin{array}{l}c<\tilde{c} \\
c<\tilde{c} \\
c<\tilde{c} \text { and } i>i_{* *}(k)\end{array}$ \\
\hline
\end{tabular}

Here $\quad u^{*}=\frac{g \beta}{\zeta \phi-\beta}, v^{*}=\frac{\rho}{\phi}\left(1-\frac{u^{*}}{c}\right)\left(u^{*}+g\right), \underset{\sim}{c}=\frac{g \beta}{\zeta \phi-\beta}, \tilde{c}=\frac{g(\zeta \phi+\beta)}{\zeta \phi-\beta}, k=\frac{h \delta}{\epsilon \theta-\delta}, \tilde{k}=\frac{h(\epsilon \theta+\delta)}{\epsilon \theta-\delta}, \underline{l}=\frac{h \delta}{\eta \psi-\delta}, i=\mu \rho c\left(1-\frac{c}{c}\right), i_{*}(k)=\left(1-\frac{k}{k}\right) \underline{l} \gamma, i^{*}=\left(\gamma+\frac{r \psi}{\theta}\right) l$, and $i_{* *}(k)$ is a complicated curve which denotes the onset of limit cycles, as discussed in Appendix A

case (ii), we observe more interesting dynamics, as shown in Fig. 2. We see that the phase-portrait of $s, x$ and $y$ is torus-shaped, indicating a quasi-periodic solution. Therefore, depending on the dynamics of the scavenger-resource system, a limit cycle in the predator-prey system can result in simple limit cycles or quasi-periodic orbits. For case (iii), Fig. 3 shows irregular and perhaps chaotic dynamics. Since $u$ and $v$ dominate the top time series, we plot a second time series for $x$ and $y$, and in this, the scavenger-resource dynamics appear irregular. Note that the scavenger and resource populations evolve on a slower timescale than the predator and prey populations. Therefore, these dynamics might easily have been overlooked.

Regarding less regular non-equilibrium dynamics, note that Rinaldi et al. (1993) and Levy et al. (2016) found chaotic behaviour in non-autonomous predator prey models.
Fig. 1 Bifurcation diagrams of the PPQRS model with $r=0.1$ and $\mu=0.1$ and $\rho=1$ (top left), $\mu=0.9$ and $\rho=0.5$ (bottom left), $\mu=0.9$ and $\rho=1$ (top right) and $\mu=0.9$ and $\rho=2$ (bottom right). The various regions are resource-free (yellow), predator- and scavenger-free (bottom left, in purple), predator-free (bottom middle, in orange), scavengerfree (middle left, in red), and the positive equilibria (middle, in blue), and non-equilibrium dynamics (right and top, in green). The labelled arrows in the bottom right diagram will be used in Section 1 to motivate our analysis of the PDE model
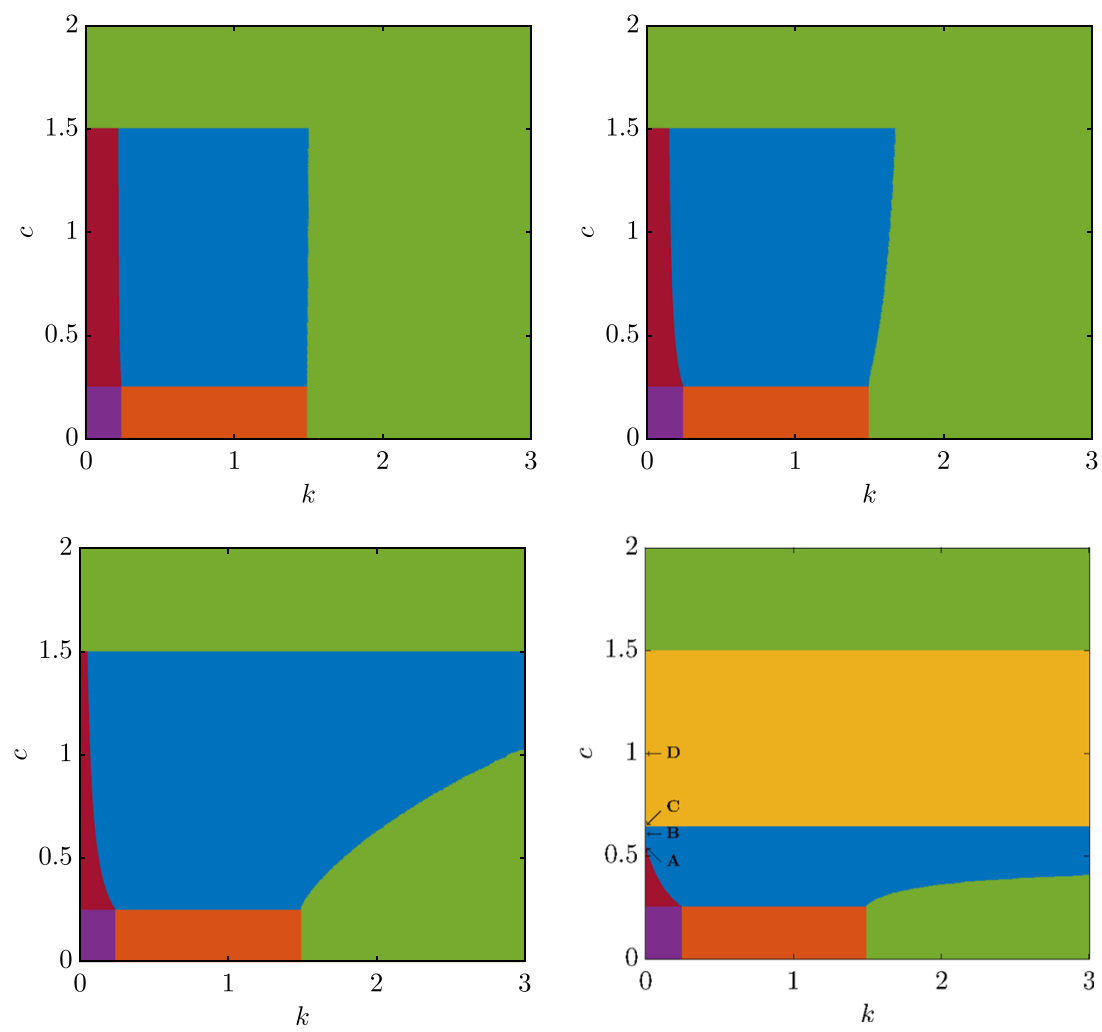

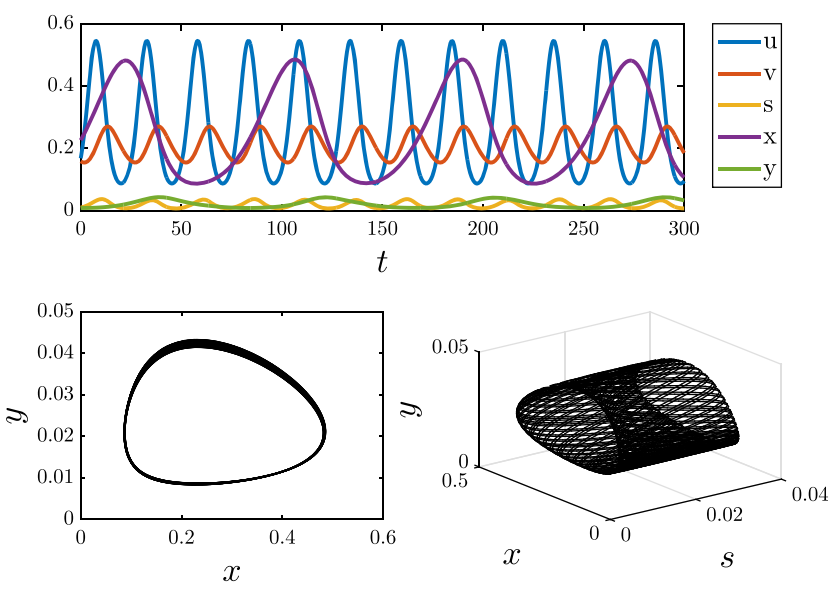

Fig. 2 Numerical solution of the PPQRS system with parameter values $r=0.1, \mu=0.1, c=1.6, k=1.6$ and $\rho=1$. We plot the phase-portraits of $x$ and $y$ and of $s, x$ and $y$ for 2500 units of time

When one parameter of a originally autonomous model was replaced by a sinusoidal forcing term, steady-state solutions became periodic solutions and periodic solutions were replaced by either quasi-periodic or, for high magnitudes of the forcing term, aperiodic solutions. Because we have two interacting limit cycles, we might expect to also find chaos in our model.

To explore if we indeed found chaos in our system, we will use a common test for chaotic behaviour: the maximal Lyapunov exponent (MLE) test. Lyapunov exponents describe the exponential rate at which a perturbation to a trajectory of a dynamical system grows or decays with time. When all Lyapunov exponents of a system are negative, we have a stable fixed point. Periodic cycles always have one Lyapunov exponent equal to zero. When we have a stable limit cycle, the other Lyapunov exponents must be negative. An $n$-period quasi-periodic solution has $n$ zero Lyapunov exponents. Finally, one or more positive Lyapunov exponents are a sign of chaotic behaviour. For a theoretical discussion of this, see Appendix B. To test for chaos in our system, we numerically approximated the largest Lyapunov exponent of our system from time series data for different
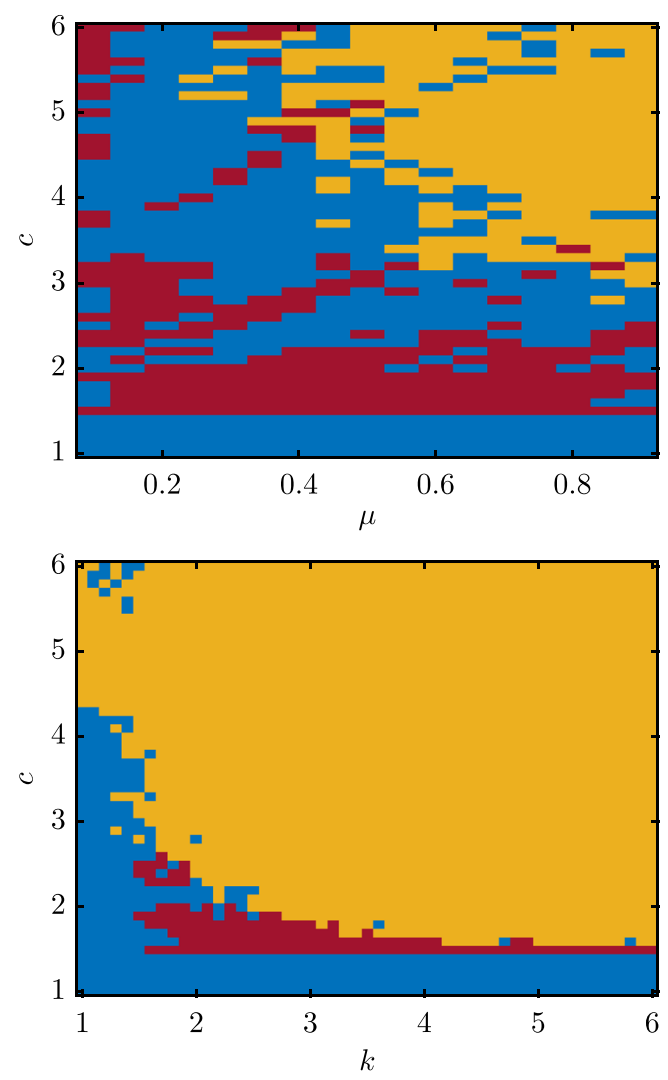

Fig. 4 Bifurcation diagrams with the red cells corresponding to at least two Lyapunov exponents with an absolute value smaller than $2 \times 10^{-3}$ (indicating quasi-periodic solutions), MLE larger than $10^{-3}$ (indicating chaos) in yellow and all MLE smaller than $10^{-3}$ (indicating steady states or limit cycles) in blue. We took parameter values $r=0.1$ and $\rho=1$, and $c=1.6, k=1.6$ and $\mu=0.9$ (when not specified)

parameter values. We used the MATLAB code as developed by Govorukhin (2004), based on techniques as described in Wolf et al. (1985) and ran the test for $t=10^{4}$ units of time.

In Fig. 4 we plot the MLE for varying $c, k$ and $\mu$. Yellow regions are predicted to be chaotic, red regions give quasi-periodic dynamics and blue regions give limit cycles or steady states. Theory tells us that a 2-torus has two Lyapunov exponents equal to zero. The red regions therefore correspond to at least two Lyapunov exponents with an
Fig. 3 Numerical solution of the PPQRS system with parameter values $r=0.1, \mu=$ $0.9, c=5, k=1.6$ and $\rho=1$. We plot the phase-portraits of $x$ and $y$ and of $s, x$ and $y$ for 2500 units of time
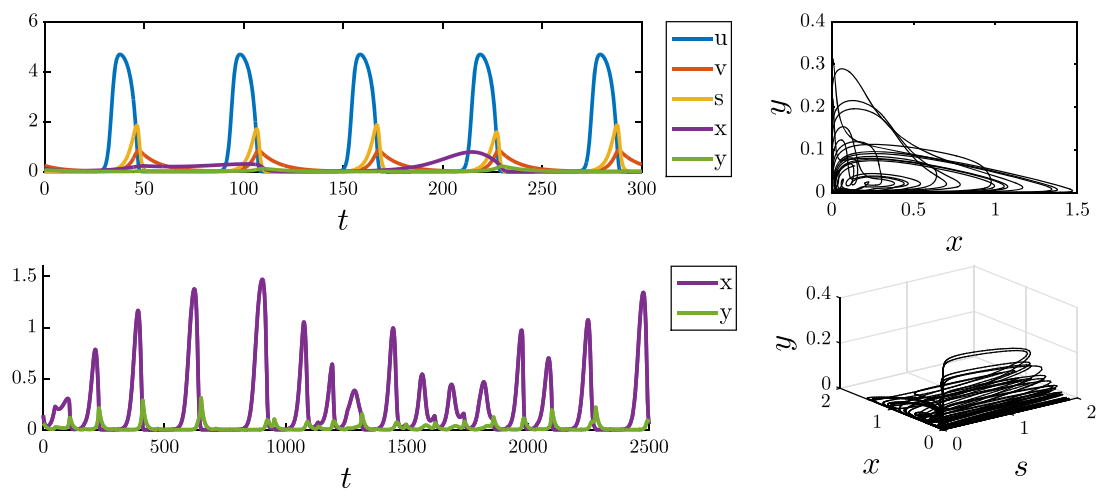
absolute value smaller than $2 \times 10^{-3}$. We know that when $c$ or $k$ are smaller than $\tilde{c}$ and $\tilde{k}$, respectively, we do not have two interacting limit cycles. Indeed, we see that below $\tilde{c}=1.5$ and $\tilde{k}=1.5$, almost no yellow values are found. When $c$ and $k$ grow above these values, the Lyapunov exponents do indicate quasi-periodic solutions, like the one as shown in Fig. 2, until the MLE grows too large, indicating chaos.

\section{PPQRS model with seasonality}

The Arctic ecosystem is strongly affected by seasonal and long-term changes. The prime reason for this is the extreme change in temperature during the year. The average air temperature in the Arctic region varies from just above $0{ }^{\circ} \mathrm{C}$ in July to around $-30{ }^{\circ} \mathrm{C}$ in January (Jones et al. 1999). Firstly, these changes in temperature affect the seal carcass availability during the year, by their influence on both the decay rate of the carrion and polar bear activity. Polar bears eat mostly whales and nargal during the summer, when there is little ice, but change their diet to seals during winter months (Roth 2003). Arctic foxes are found to obtain almost half their intake of protein from marine foods during these winter months, when the lemming population is low (Roth 2002). The lemming population is also known to oscillate over a longer timescale, with sharp peaks every 3 to 4 years (Fuller et al. 1975). These oscillations too are reflected in the population dynamics and food choice of Arctic foxes; in Roth (2003), a strong correlation was found between the body mass of polar bears and the population size of the Arctic foxes in years with little lemmings, and no correlation was found in years with many lemmings available for the foxes. As the body mass of a polar bear depends on the amount of seals it has caught, this suggests that in winters with a low lemming population, marine food sources are a much more important part of the Arctic fox's diet.

We use the same approach as Levy et al. (2016) (who studied a non-autonomous generalization of the PPS model in order to understand the role of seasonality in modifying predator-prey-subsidy dynamics) and replace the constants $\delta, \rho, \beta, r$ and $\gamma$ by the following time-dependent parameters:

$\delta(t)=\delta_{0}\left(1+\delta_{1} \sin \left(\frac{2 \pi t}{\tau}\right)\right)\left(1+\delta_{2} \sin \left(\frac{\pi t}{2 \tau}\right)\right)$,

$\rho(t)=\rho_{0}\left(1-\rho_{1} \sin \left(\frac{2 \pi t}{\tau}\right)\right)$,

$\beta(t)=\beta_{0}\left(1+\beta_{1} \sin \left(\frac{2 \pi t}{\tau}\right)\right)$,

$r(t)=r_{0}\left(1-r_{1} \sin \left(\frac{2 \pi t}{\tau}\right)\right)$, $\gamma(t)=\frac{\gamma_{0}}{e^{\gamma_{1} T_{0}}-1}\left(e^{\gamma_{1} T(t)}-1\right)$

where

$T(t)=T_{0}-A_{0} \sin \left(\frac{2 \pi t}{\tau}\right)$

Here, $\tau$ is equal to the length of a year. We obtained the equations for $\rho(t)$ and $c(t)$ by multiplying the growth rate of the seals $\rho x\left(1-\frac{x}{c}\right)$ by a seasonal term:

$\rho_{0}\left(1-\rho_{1} \sin \frac{2 \pi}{\tau}\right) x\left(1-\frac{x}{c_{0}}\right)$.

The constant $\rho_{0}$ is the average yearly rate of natural increase of the prey. Note that if we take $\rho_{1}=0$, we get back our autonomous rate of growth (Turchin and Hanski 1997). We have chosen for a simple sinusoidal form of our oscillations, following (Levy et al. 2016) and (Turchin and Hanski 1997). We see that $\rho(t)$ has a minimum at $t=\frac{1}{4} \tau$. Consequently, mid-winter occurs at $t=\frac{1}{4} \tau$ in our model, as we assume that the population growth of seals is the lowest in the winter.

We assume that the polar bear and lemming populations grow the least in the winter as well, which leads to a similar expression for $r(t)$. The constant $\beta_{0}$ is the average death rate of the bears and the constant $\delta_{0}$ is the average death rate of the foxes. We assume that the most foxes and bears die in winter, which is why we multiplied $\beta_{0}$ and $\delta_{0}$ by $\left(1+\beta_{1} \sin \left(\frac{2 \pi t}{\tau}\right)\right)$ and $\left(1+\delta_{1} \sin \left(\frac{2 \pi t}{\tau}\right)\right)$, respectively. On top of this, polar foxes are known to migrate every 3 to 4 years (Wrigley and Hatch 1976). This migrating is a second cause of oscillations in the mortality rate, because of the lack of food, increased risk of disease and risk of getting trapped by hunters during the migration (Levy et al. 2016; Wrigley and Hatch 1976). We incorporated those effects of 4-yearly migration in our model by multiplying our expression for $\delta(t)$ with $\left(1+\delta_{2} \sin \left(\frac{\pi t}{2 \tau}\right)\right)$. Finally, we expect that the decay rate of the seal carrion is dependent on the temperature. We assume that the temperature takes the form $T(t)=T_{0}-A_{0} \sin \left(\frac{2 \pi t}{\tau}\right)$, where $T_{0}$ is the average temperature and $A_{0}$ the amplitude of the yearly fluctuations in temperature, and we assume that $t=n / 365$ with $n$ the number of passed days. We take the decay rate of the seal carrion to be

$\gamma(t)=\frac{\gamma_{0}}{e^{\gamma_{1} T_{0}}-1}\left(e^{\gamma_{1} T(t)}-1\right)$,

where $\gamma_{0}$ is the reference decay rate and $\gamma_{1} \neq 0$ a scaling parameter. Note that when we take $A_{0}=0$, we have $T(t)=$ $T_{0}$ and $\gamma(t)=\gamma_{0}$. 
With this, our non-autonomous model reads

$\frac{d u}{d t}=\rho(t) u\left(1-\frac{u}{c}\right)-\phi\left(\frac{u v}{u+g}\right)$,

$\frac{d v}{d t}=\left(\frac{\zeta \phi u}{u+g}\right) v-\beta(t) v$,

$\frac{d s}{d t}=\mu \phi\left(\frac{u v}{u+g}\right)-\gamma(t) s-\psi\left(\frac{s y}{x+s+h}\right)$,

$\frac{d x}{d t}=r(t) x\left(1-\frac{x}{k}\right)-\theta\left(\frac{x y}{x+s+h}\right)$,

$\frac{d y}{d t}=\left(\frac{\epsilon \theta x+\eta \psi s}{x+s+h}\right) y-\delta(t) y$.

Since Levy et al. (2016) already considered a systematic study of what is essentially the decoupled form of Eqs. 5.35.5 , we shall be most interested in the role of seasonality in Eqs. 5.1-5.2 and then the influence of this has on modifying the resulting dynamics of Eqs. 5.3-5.5. As such, we shall focus on non-autonomous form of these equations using $\rho(t)$ and $\beta(t)$. We also ran simulations using $r(t), \gamma(t)$ and $\delta(t)$, yet the resulting dynamics were akin to those in Levy et al. (2016) and will not be repeated here.

\section{Effect of parameter oscillations on steady states}

We fix all parameters except $c_{0}$ and $k_{0}$ to correspond to Fig. 1 (lower right panel). $T_{0}=258 \mathrm{~K}$ is the average Arctic temperature (Jones et al. 1999). By varying $k$ and $c$, we can fix our solutions to one of the five possible steady states: the resource-free, predator- and scavengerfree, predator-free, scavenger-free, or positive equilibrium. We start with $k=1$ and $c=0.5$, so that we are in the positive equilibrium area. We varied $\rho_{1}$ and $\beta_{1}$ and plot the resulting time series for $x$ and $y$ in Figs. 5 and 6 , respectively. An increase in either parameter is seen to increase the amplitude of boom and bust dynamics, and to make such dynamics more intermittent and less regular in time.

We see that there are a few cases where the oscillations push the resources or scavengers to extinction and one case where oscillations are necessary for the scavengers to survive. We found cases were the scavengers went extinct as a result of non-autonomous oscillations in $\beta(t)$, the death rate of the predators. Remarkably, this $\beta_{1}$ turned out to be necessary for the survival of the scavengers in another case, which we plot in Fig. 7 for various $\beta_{1}$. This demonstrates that the predator-prey dynamics can have a great influence on the resource and scavenger populations; particularly in fragile ecosystems that are in a state that is close to a bifurcation, changes in seasonal variation can bring the preservation of species in danger.
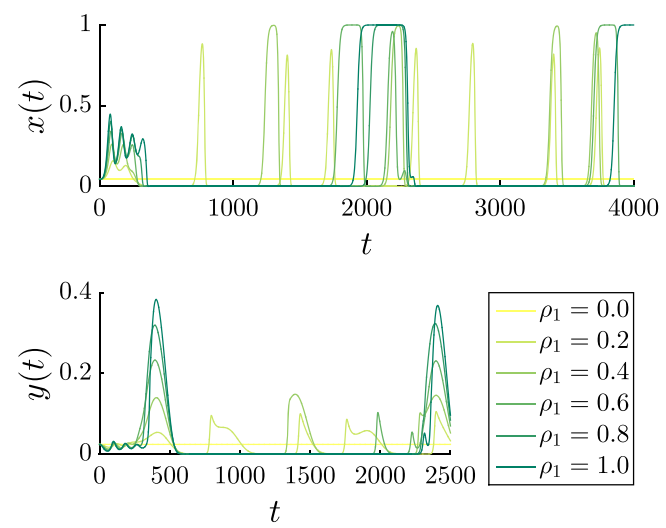

Fig. 5 Time series for the non-autonomous model (5.1)-(5.5) given varying $\rho_{1}$ with parameter values $k=1, c=0.5, \mu=0.9, \rho_{0}=$ $2, r_{0}=0.1, \gamma_{0}=1, \delta_{0}=0.1, \beta_{0}=0.1, \gamma_{1}=1, T_{0}=258, \tau=500$ and $\rho_{1}=r_{1}=\delta_{1}=\delta_{2}=\beta_{1}=A_{0}=0$
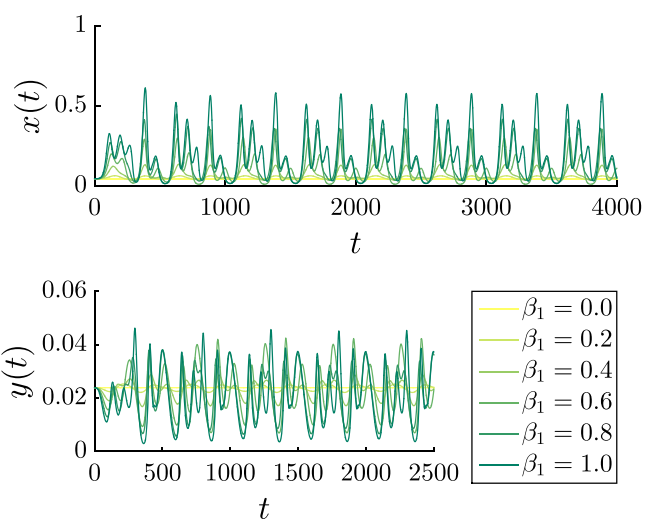

Fig. 6 Time series for the non-autonomous model (5.1)-(5.5) given varying $\beta_{1}$ with parameter values $k=1, c=0.5, \mu=0.9, \rho_{0}=$ $2, r_{0}=0.1, \gamma_{0}=1, \delta_{0}=0.1, \beta_{0}=0.1, \gamma_{1}=1, T_{0}=258, \tau=500$ and $\rho_{1}=r_{1}=\delta_{1}=\delta_{2}=\beta_{1}=A_{0}=0$

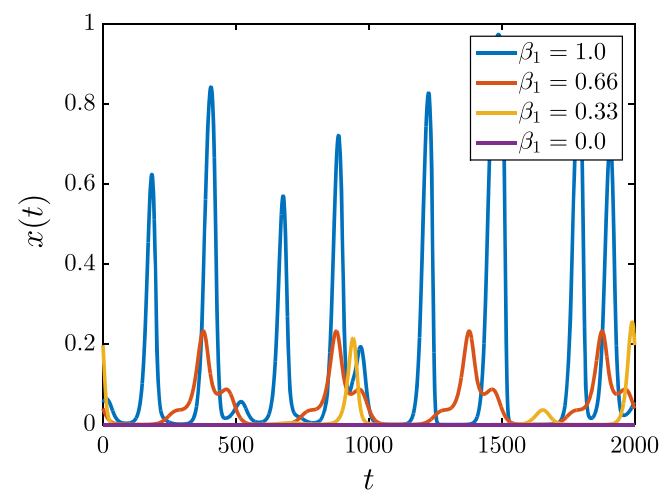

Fig. 7 Time series for various choices of $\beta_{1}$. We take the parameter values $k=1, c=0.65, \mu=0.9, \rho_{0}=2, r_{0}=0.1, \gamma_{0}=1, \delta_{0}=$ $0.1, \beta_{0}=0.1, \gamma_{1}=1, T_{0}=258, \tau=500$ and $\rho_{1}=r_{1}=\delta_{1}=\delta_{2}=$ $A_{0}=0$ 


\section{Effect of parameter oscillations on periodic, quasi-periodic and aperiodic solutions}

We investigate the effects of non-autonomous terms on the periodic, quasi-periodic and aperiodic solutions of our system. Again, we examine one time-dependent parameter at a time. We vary the amplitude of the oscillating parameter between 0 and 1 and vary the period by taking $\tau=1.15^{i}$, for $i=1,2,3 \ldots 100$. By varying $\tau$, we change the relative timescale between the oscillating parameter and the natural oscillations of the system.

We give sample bifurcation diagrams of the MLE in Fig. 8. Depending on the parameter regime selected, quasiperiodic or chaotic dynamics can either be rare or ubiquitous in parameter space, highlighting the sensitivity of the system on the carrying capacities $c$ and $k$.

An interesting observation is that there are stability regions in the bifurcation diagrams, in spite of nonautonomous seasonal forcing. Indeed, for some cases, introducing a non-autonomous seasonal forcing term actually stabilizes non-equilibrium dynamics. As an example, we plot time series of the solution for $\delta_{1}=0$ (the autonomous case), and for $\delta_{1}=1$, with various $\tau$, in Fig. 9. For $\tau=1.15^{50}$ and $\delta_{1}=1$, we find that the non-autonomous oscillations drive the resource to extinction.

\section{Extension to a spatial PDE model}

In the Arctic, the seal carcasses are spatially separated from the lemmings. Lemmings live on the land, while the seals are caught by bears on the ice. To investigate the effects of this spatial separation, we propose a continuum partial differential equation (PDE) model:

$\frac{d u}{d t}=d_{1} \Delta u+\rho u\left(1-\frac{u}{c}\right)-\phi\left(\frac{u v}{u+g}\right)$, in $\Omega_{2}$,

$\frac{d v}{d t}=d_{2} \Delta v+\left(\frac{\zeta \phi u}{u+g}\right) v-\beta v$, in $\Omega_{2}$,

$\frac{d s}{d t}=\mu \phi\left(\frac{u v}{u+g}\right)-\gamma s-\psi\left(\frac{s y}{x+s+h}\right)$, in $\Omega_{2}$,

$\frac{d x}{d t}=d_{4} \Delta x+r x\left(1-\frac{x}{k}\right)-\theta\left(\frac{x y}{x+s+h}\right)$, in $\Omega_{1}$,

$\frac{d y}{d t}=d_{5} \Delta y+\left(\frac{\epsilon \theta x+\eta \psi s}{x+s+h}\right) y-\delta y$, in $\Omega_{1} \cup \Omega_{2}$.

This model is based on the model proposed in Bassett et al. (2017) for PPS dynamics and hence, is a suitable spatial extension of the PPQRS model. We consider a circular, two-dimensional domain $\Omega_{1}$ on which the resources live, surrounded by a ring-shaped domain $\Omega_{2}$ for the prey, predators and quarry. On $\Omega_{1}, s=0$ and on $\Omega_{2}, x=0$. The scavengers are assumed to move freely between the two domains. We take no-flux boundary conditions and assume that the carcasses are not able to move. The bears, seals, foxes and lemmings are assumed to move at rates $d_{1}, d_{2}, d_{4}$ and $d_{5}$, respectively.

Before considering our full PPQRS system, we note that in Medvinsky et al. (2002), a spatial Rosenzweig-Macarthur model, equivalent to our Eqs. 6.1 and 6.2, was examined on a rectangular domain. There, two cases were considered:
Fig. 8 Bifurcation diagrams of the positive MLE. Red cells correspond to quasi-periodic solutions, yellow cells indicate chaos and blue cells indicate steady states or limit cycles. We take $c=1.6, k=0.3$ for the two panels, $c=4.8, k=2.4$ for the lower left panel and $c=1.6, k=1.6$ for the lower right panel. Other parameter values are as follows: $r=$ $0.1, \gamma_{0}=1, \delta_{0}=0.1, \rho=1$, $\beta_{0}=0.1, \mu=0.1, T_{0}=258$ and $\rho_{1}=r_{1}=\delta_{1}=\delta_{2}=\beta_{1}=$ $A_{0}=0$ (when not specified)
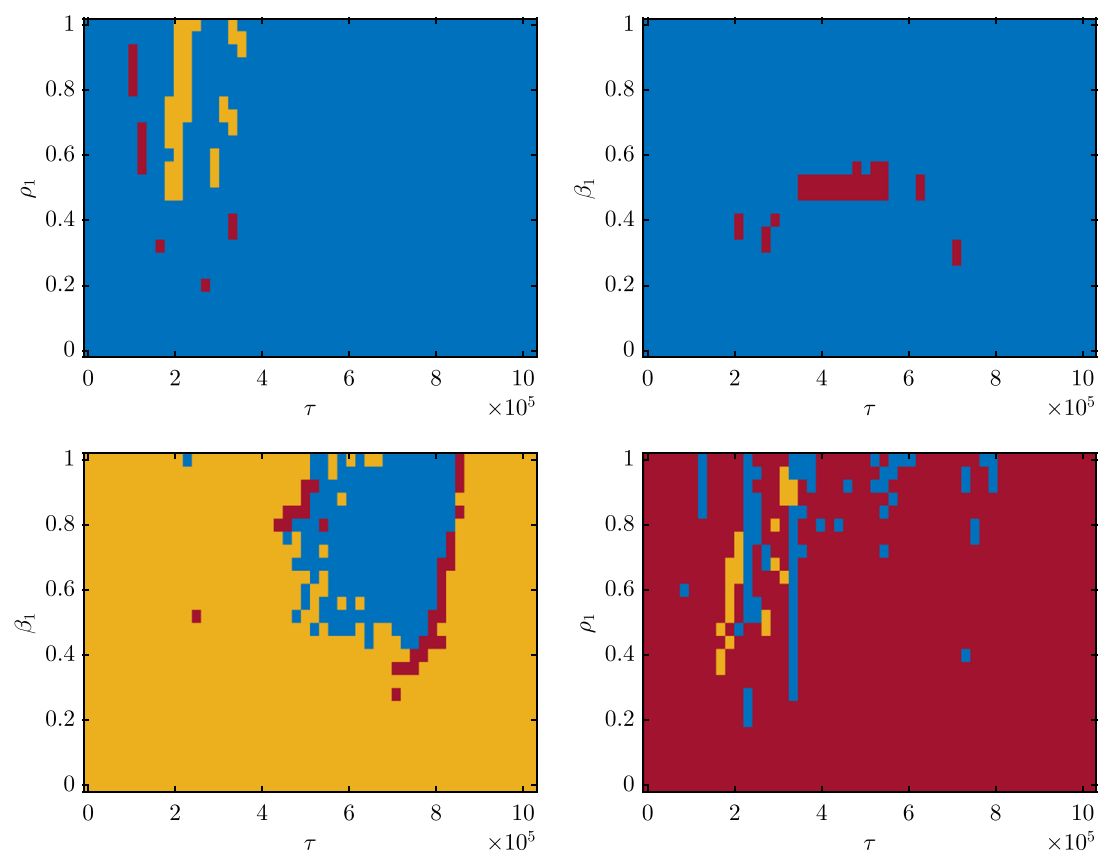
Fig. 9 Time series of the resource $x(t)$ with varying $\delta_{1}$ and $\tau$. We took parameter values $r=0.1, \gamma_{0}=1, \delta_{0}=0.1, \rho=$ $1, \beta_{0}=0.1, \mu=0.9, c=$ 4.8, $k=2.4, T_{0}=258$ and $\rho_{1}=r_{1}=\delta_{2}=\beta_{1}=A_{0}=0$

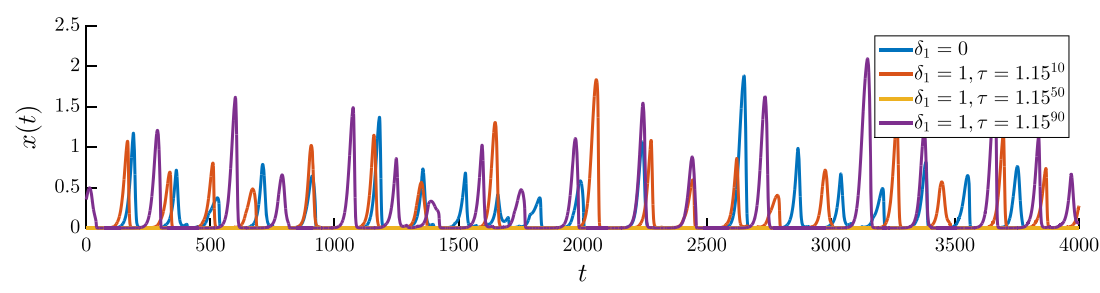

$c<\tilde{c}$, the stationary case, and $c>\tilde{c}$, the limit cycle case. When $c<\tilde{c}$, the system approaches a spatially homogeneous steady state that is equal to the ODE solution: $u=u^{*}$ and $v=v^{*}$. When $c>\tilde{c}$, for a not too weakly perturbed initial distribution, a jagged spatial pattern was found that is persistent in time (Medvinsky et al. 2002, p. 329).

\section{Simulation method and conditions}

We used the finite element solver of COMSOL Multiphysics. We consider a circular domain $\Omega \subset \mathbb{R}^{2}$ of radius 500 units of length, centred around $(0,0)$, which we divide in two areas $\Omega_{1}$ and $\Omega_{2}$. The inner domain $\Omega_{1}$ is also circular and centred around $(0,0)$, with a radius of 250 units of length. In our motivating example, this would be the land on which the lemmings live. The outer domain $\Omega_{2}$ is an annular region, inhabited by the seals and bears. The foxes are assumed to be able to move freely over the whole domain $\Omega=\Omega_{1} \cup \Omega_{2}$. Further, at first, we assume that the seals, bears, foxes and lemmings all diffuse at the same rate $d=d_{1}=d_{2}=d_{4}=d_{5}$. The carcasses cannot move (so, $d_{3}=0$ ). This is because, although systems of two reaction-diffusion equations usually need different diffusivities in order to exhibit the Turing instability and resulting pattern formation, higher-order systems can have less regularity and hence, in some cases, parameter restrictions can be less restrictive yet Turing instabilities can still be found. Therefore, it is sensible to start with all non-trivial diffusivities the same, if one is searching for patterns in our spatial model. A comprehensive study of the spatial system with different diffusivities, in order to classify the various routes to the Turing instability and hence to spatial patterning, could be an interesting direction for future work.

As initial conditions for $u, v, x$ and $y$, we take a random perturbation in both spatial directions of $u^{*}, v^{*}, x^{*}$ and $y^{*}$, where $u^{*}$ and $v^{*}$ are defined in Eq. A.7 and

$x^{*}=\frac{h \delta}{\epsilon \theta-\delta}, \quad y^{*}=\frac{r}{\theta}\left(1-\frac{x^{*}}{k}\right)\left(x^{*}+h\right)$.

Those are the equilibrium values of the scavengers and resources if no quarry is present. We take $s(0)=0$. Note that we presently assume that the scavengers do not have a preference to one of the two domains and have equal access to every part of the region. As an extension we might for example consider the case where the scavengers would only move to the outer domain when the number of resources is below a certain threshold.

\section{Comparison between PDE and ODE dynamics}

We will take parameter values as in Fig. $1 \mathrm{~d}: k=1.6, \mu=$ $0.9, \rho=2, r=0.1$ and $d=1$. We take those values because the resource-free equilibrium is stable for certain values of $c$. We take consecutively $c=0.55$, such that $i<i_{*}(0), c=0.60$, such that $i_{*}(0)<i<i *, c=0.65$, such that $i>i *$ and $c=1$, such that $i \gg i^{*}$. We plot spatial dynamics for the scavenger $y$ in Fig. 10. These choices of parameter regimes correspond to the labels given in Fig. 1d: $\mathbf{A}$, in the red (scavenger free) region for $c=0.55, \mathbf{B}$, in the blue (positive equilibrium) region for $c=0.60, \mathbf{C}$, just in the yellow (resource-free) region for $c=0.65$ and $\mathbf{D}$, in the yellow (resource-free) region for $c=1$.

We see that as $i$ increases, the number of scavengers on the outer ring increases. While in the ODE model, the resources would not have survived for $c=0.65$ (see yellow region in Fig. 1d), in the spatial PDE model, they do survive. When we increase to $c=1$, we see that the oscillations of the resources and scavengers disappear. We find that $x \rightarrow x^{*}$ and $y \rightarrow y^{*}$ for all $x$ and $y$ on the circle. So, we found a spatially uniform positive equilibrium, even though the ODE model would give a resource-free equilibrium area (yellow region in Fig. 1d). Hence, spatial dispersion has allowed both populations to persist.

When we the value of $c$ further, so that the prey and predator are in a limit cycle (green region in Fig. 1), we see that we obtain a spatial non-homogeneous pattern of scavengers on both the inner circle and the outer ring (see Fig. 11a). For this case, we also plot select time series in Fig. 12. In particular, we plot time series at a single point in the domain, for the population averaged over the domain, and also for the ODE model solution with the same parameter values. While populations in individual locations appear to oscillate with period different to the ODE model, the spatially averaged plot appears to oscillate with a period close to that of the ODE model (approximately 14.6 time units).

We explored the chaotic case by increasing $c$ and $k$ even further. We plot a pattern-rich solutions of $y$ at $t=5000$ in Fig. 11b. The corresponding MLE is 0.012 , and we likely have spatio-temporal chaos. We plot corresponding time series in Fig. 13, again showing time series for a single 
Fig. 10 The scavenger population $y$ for $t=5000$ with parameter values $k=1.6, \mu=$ $0.9, \rho=2, r=0.1$ and $d=1$ and $\mathbf{a} c=0.55, \mathbf{b} c=0.60$, c $c=0.65$ and $\mathbf{d} c=1$. Note the smoothing of the spatial dynamics for the scavenger $(y)$ due to the increase in $c$, which in turn increases the quarry

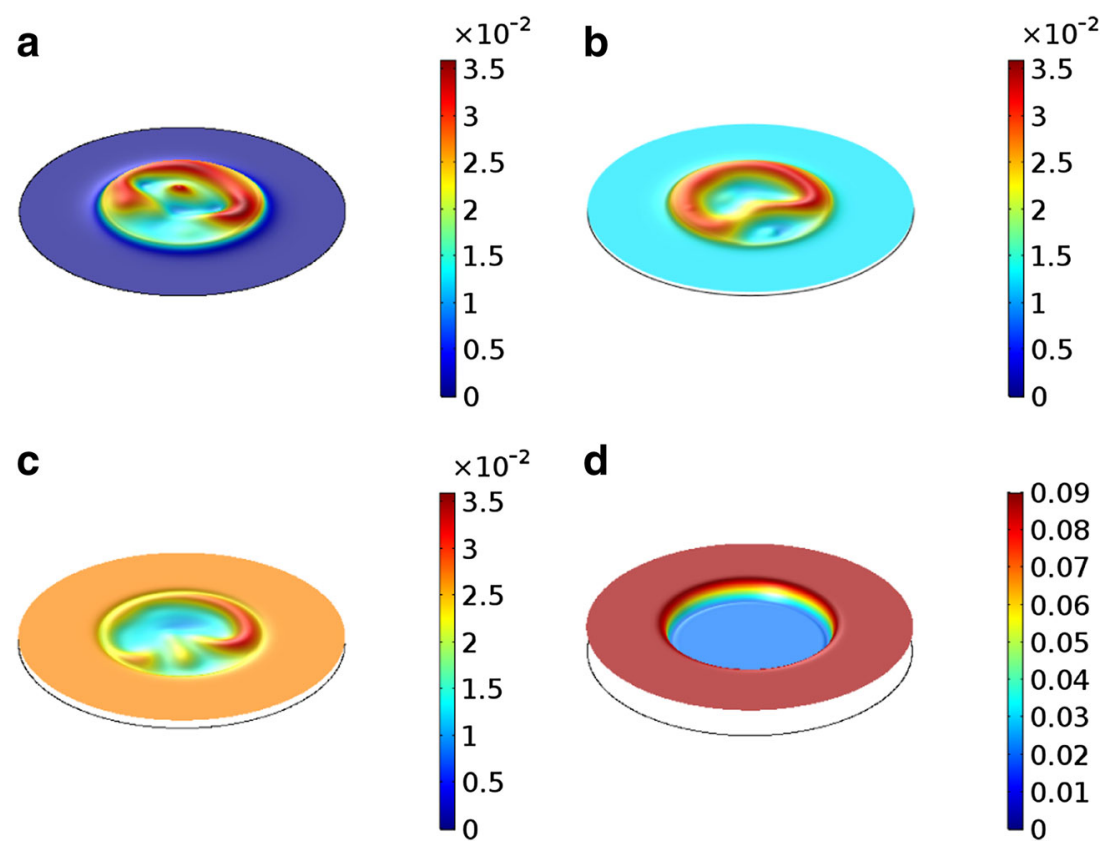

point in the domain, the spatially averaged population in the domain and the ODE model solution. It is interesting to see that the average number of scavengers is almost constant and is never close to zero. Clearly, the spatial model gives a different solution from the ODE case. In particular, while oscillations are present in the average population, the oscillations are small enough so that the population is ever close to extinction. In this manner, the spatial diffusion regularizes the solutions and resolved the paradox-of-enrichment in this setting.

Similar behaviour was observed for a spatial Rosenzweig-Macarthur model (Petrovskii et al. 2004). It is well known that in the ODE Rosenzweig-Macarthur model, for large values of $c$, both the predator and prey populations are very small for most of the time and might easily go extinct. This effect is known as the 'paradox of enrichment' and was first described by Rosenzweig (1971).

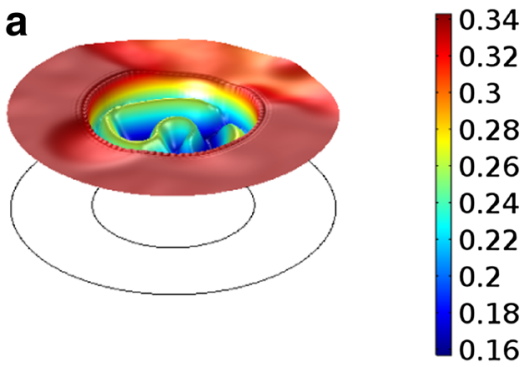

Fig. 11 The scavenger population $y$ for $t=5000$ with parameter values $\mu=0.9, \rho=3, r=1, d=1$ and $\mathbf{a} c=1.6, k=1.6$ and $\mathbf{b}$ $c=3.5, k=2.5$. Larger carrying capacity results in more drastic spatial variation, as might be expected from the paradox of enrichment. However, unlike for an ODE model, here, there are always regions of
In Petrovskii et al. (2004), it was observed that the addition of spatial diffusion can resolve this paradox, as the amplitude of the oscillations stays small in the spatial PDE case, preventing extinction. This is exactly what we observe for the more complicated PPQRS model when spatial diffusion is permitted.

\section{Discussion and biological implications}

When analysing the PPQRS model, we found that its steadystate behaviour was equivalent to the PPS model that was studied by Nevai and Van Gorder (2012). Therefore, we will only look at the periodic, quasi-period and aperiodic solutions. We will compare our results with the PPS model that was studied by Nevai and Van Gorder (2012) and will compare them with the non-autonomous PPS system with

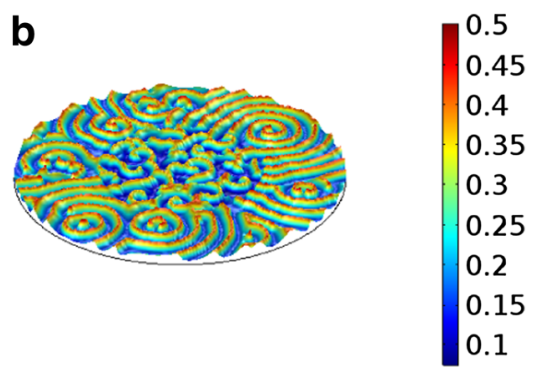

large concentration for all time, and hence, the boom and bust dynamics are highly localized, with the overall population remaining safe from extinction even though local extinction on small timescales could be possible 
Fig. 12 Time series of $y$ for a the local population at the point $(125,0), \mathbf{b}$ the spatial average of $y$ and $\mathbf{c}$ the ODE solution of $y$.

Parameter values are

$k=1.6, c=1.6, \mu=0.9, \rho=$ $3, r=1$, and $d=1$ for $\mathbf{a}, \mathbf{b}$ and $d=0$ for $\mathbf{c}$

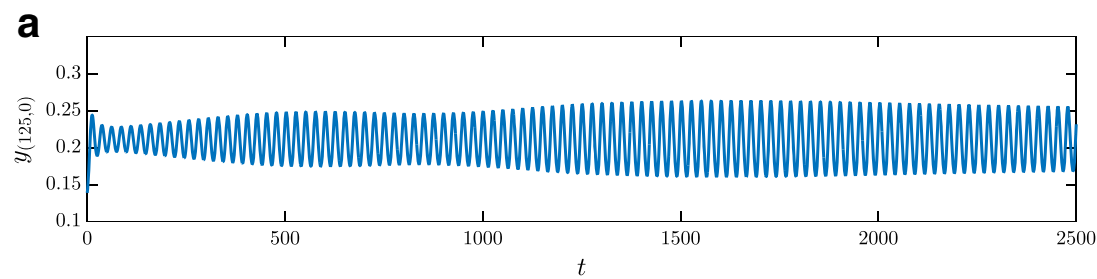

b

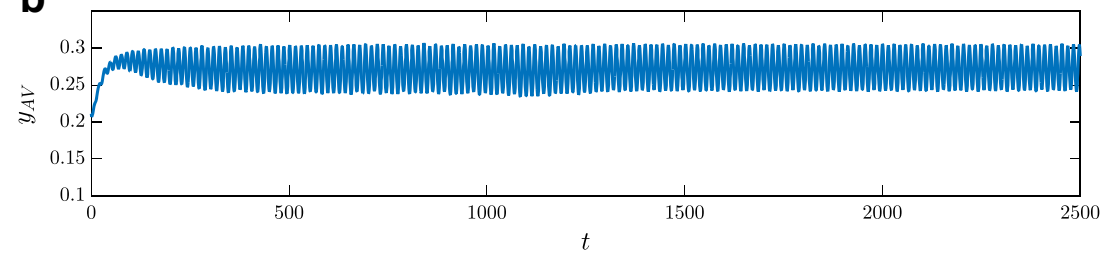

C

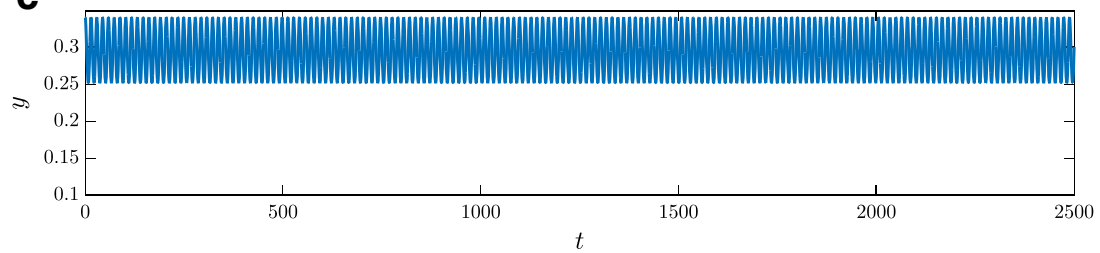

varying input rate that was studied by Levy et al. (2016). We will also briefly compare our PPQRS model with an apparent competition model and compare the ODE and PDE models.

\section{Non-equilibrium solutions}

When $c>\tilde{c}$, the predator-prey system is in a limit cycle and the input rate $i$ of quarry is therefore not constant, but given by

$i(t)=\mu \phi \frac{u(t) v(t)}{u(t)+g}$.
In Fig. 14, we plot the non-constant input rate $i(t)$ for various values of $c$. When also $k>\tilde{k}$, we have two interacting limit cycles. When $c$ is just larger than $\tilde{c}$, we see in Fig. 14 that the input rate has a sinusoidal shape. In Levy et al. (2016), the non-autonomous PPS model was studied, with $i$ replaced by

$i(t)=0.05\left(1+i_{1} \cos \left(\frac{2 \pi t}{\tau}\right)\right)$.

When the parameters $i_{1}$ and $\tau$ were varied, the largest MLE that was found had a value of 0.0012 , for $i_{1}=\frac{14}{15}$ and
Fig. 13 Time series of $y$ for a the local population at the point $(125,0), \mathbf{b}$ the spatial average of $y$ and $\mathbf{c}$ the ODE solution of $y$. Parameter values are

$k=2.5, c=3.5, \mu=0.9, \rho=$ $3, r=1$ and $d=1$ for $\mathbf{a}, \mathbf{b}$ and $d=0$ for $\mathbf{c}$

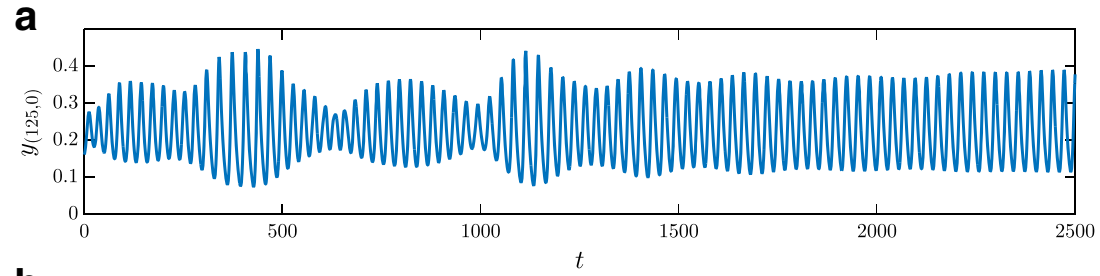

b
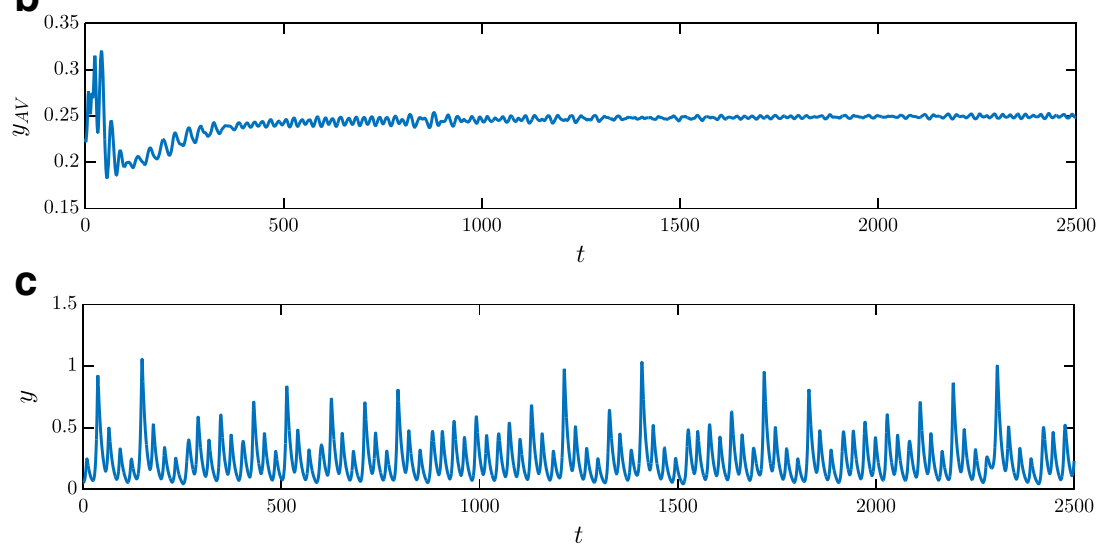


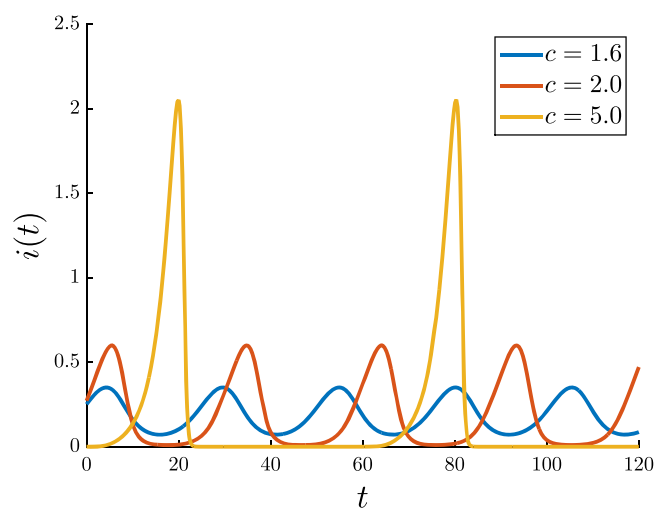

Fig. 14 Time series of the input rate $i(t)$ against time for $c=1.6,2.0$ and 5.0 with $\rho=1$

$\tau=1.15^{28} \approx 50$ and further $r=0.1$ and $k=2$. When we further increase $c$ in the PPQRS model, the input rate function approaches zero, with sharp peaks every once in a while. This causes the scavenger-resource dynamics to become chaotic (see Fig. 3 for an example). Note that the scavenger and resource populations evolve on a slower timescale than the predator and prey populations. Therefore, the chaotic dynamics might easily be overlooked.

It is interesting to see that we can find chaotic solutions in the PPQRS model without increasing $k$, where the PPS model with a sinusoidal time varying input rate and $k=2$ does not give chaos. We suspect that it is the peaked form of the input rate function for larger $c$ that causes the chaotic behaviour of our solution. In biological terms, this indicates that if for most of the time, there are no carcasses, but if there is quarry, it comes in large amounts; this can push a sinusoidal limit cycle of foxes and lemmings, where $k$ is just larger than $\tilde{k}$, in to chaos. As bears are known to only eat seals during a short period of time in the year (Roth 2003), a 'peak-like' input rate like the one for $c=5.0$ in Fig. 14 is probably more realistic than the sinusoidal one considered in Levy et al. (2016).

\section{Comparison with autonomous and non-autonomous PPS models}

The main difference between our PPQRS model given by Eqs. 2.1-2.5 and the PPS model (Nevai and Van Gorder 2012) is the addition of an extra limit cycle of prey and predators to the system. Because of this limit cycle, we found that in certain cases, the resource-free equilibrium is not stable for any value of $c$ or $k$, whereas in the PPS model of Nevai and Van Gorder (2012), this equilibrium can always be made stable. The existence of a second limit cycle results in the occurrence of quasi-periodic and aperiodic solutions, which were not found in the PPS model of Nevai and Van Gorder (2012). Finally, in Levy et al. (2016), a nonautonomous PPS model was studied, with a non-constant input rate of the form Eq. 7.2. This oscillating input rate did interact with the existing limit cycle of the scavengers and resources, resulting in quasi-periodic solutions. Unlike for our autonomous PPQRS model, no aperiodic solutions were found for this non-autonomous PPS model.

Including seasonality in our model, as done in Section 8, can fundamentally change the solutions of the autonomous system, turning equilibria into periodic solutions and periodic solutions into quasi-periodic and even aperiodic solutions. In one case, the addition of a non-constant death rate of the predators $\beta(t)$ was actually found to be necessary for the scavengers to persist. Our findings show that the predator dynamics can have a great influence on the resource and scavenger populations. Especially in fragile systems, that are in a state that is close to a bifurcation, changes in seasonal variation can bring the preservation of species in danger. Although part of these results could have been found for non-autonomous PP and PPS models as well, we have not found them in the literature.

\section{Comparison with apparent competition models}

As we noted before, our system has similarities with an apparent competition model, consisting of two resources and one consumer. While the two resources do not compete directly, they do have a negative effect on each other through their shared predator: they are in 'apparent' competition. In our model, the $\mathrm{Q}$ and $\mathrm{R}$ would be the resources and the $\mathrm{S}$ the consumer. In both type of models, we can find steadystate solutions, limit cycles and chaos (Abrams et al. 1998). Our resource and quarry are also in apparent competition. Recall for instance that an increase in the amount of quarry can push the resource in extinction.

A difference between the second resource in an apparent competition model and the quarry in the PPQRS model is the independence of the growth rate of the quarry on the amount of quarry already present. When only quarry and one consumer are present in the PPQRS model, the system will tend either to a quarry only equilibrium or to an equilibrium where both consumer and quarry coexist. This two-species subsystem does not exhibit limit cycle solutions (Nevai and Van Gorder 2012). However, when we add the predator prey system, we can let the consumer quarry system oscillate due to an externally driven oscillation in the growth rate of the quarry. So, both in the apparent competition model and the PPQRS model, we can have two sources of oscillations. In both models, the first is the limit cycle that arises from the consumer (scavenger) and resource dynamics. The second one is different in both models. For the apparent competition model, the second cycle arises again from intra specific interaction dynamics. In the PPQRS model, the second cycle arises by an external forcing term of the growth rate of the quarry, which is why we compared our 
model with the non-autonomous model as studied in Nevai and Van Gorder (2012).

\section{Comparison between ODE and PDE models}

We found just as in the ODE model that an abundance of quarry can push the scavenger-resource dynamics to a homogeneous steady state that is constant in time. Further, we found that in order for scavengers to enter the predatorprey-quarry area, there needs to be enough quarry available for the scavengers to survive on it alone, without having to depend on resources as well. This result is especially important for larger areas, where it takes some time for scavengers to travel from an area of resources to an area of quarry. Arctic foxes are known to migrate every 3-4 years, as a result of a decline in the lemming population (Wrigley and Hatch 1976, p. 149). On such a timescale, we would expect this to have an influence on our model. When the amount of quarry is too low for scavengers to enter the quarry-rich area, this does not mean that the existence of quarry has no influence on our system. Scavengers can live close to the border of the two areas, eating both quarry and resources. We saw that by decreasing the amount of quarry available, we could alter the dynamics of the scavengers and resources from a positive steady state to a limit cycle.

Finally, in the ODE model, we found chaotic behaviour for large carrying capacities $c$ and $k$. The population sizes tend to oscillate wildly, with sharp peaks and valleys. In the PDE model, we see that the average population sizes do actually vary less when the carrying capacities are increased. So, increasing $c$ turns out to be stabilizing instead of destabilizing when animals can move around. When a predator has eaten all prey in a certain area, it can just move on to the area next to it. But when migration is not longer possible, this might ultimately drive the predator to extinction. This could happen when the living area of a predator decreases, for example because of melting ice.

\section{Biological implications}

Biologically, it is perhaps most interesting to consider how the interaction between the predator-prey system and the resource-scavenger-quarry system will modify the stability of individual equilibrium or non-equilibrium states. We exhibit a rich variety of dynamics in the PPQRS model (2.1)-(2.5), corresponding to resource-free equilibrium, predator- and scavenger-free equilibrium, predator -free equilibrium, scavenger-free equilibrium, positive equilibrium values, as well as non-equilibrium dynamics consisting of simple limit cycles and less regular dynamics such as chaos. The destabilization of any such state as well as the transition between states is of strong importance to the well-being of any particular population.
We find that the conversion factor, $\mu$, as well as the individual carrying capacities $c$ and $k$ of the prey and resource, respectively, are useful bifurcation parameters. As either is made sufficiently large, we can often observe a transition from equilibrium to non-equilibrium dynamics such as limit cycles. In cases where limit cycles are already present, an increase in either of these parameters may push the system into chaotic dynamics. As limit cycles are a proxy for boomand-bust dynamics, within which one or more populations may become close to extinction, understanding when such cycles are possible is important for sake of conservation efforts.

When the predator-prey population is in equilibrium, we tend to observe equilibrium or limit cycle dynamics in the resource-scavenger-quarry system. A change in equilibrium value of the predator-prey system is sufficient to destabilize a positive equilibrium in the resource-scavenger-quarry system and push it into boom-and-bust dynamics. Therefore, while the predator-prey system is decoupled from the other dynamics, its behaviour strongly determines the behaviour of the resource-scavenger-quarry system, depending on how reliant the scavenger is on the quarry input from the predator-prey system.

In the case where the predator-prey system exhibits limit cycles, these dynamics can destabilize those of the resource-scavenger-quarry system, resulting in limit cycles or even less regular dynamics such as chaotic fluctuations in population of the scavenger and the resource. Hence, the stability of the resource-scavenger-quarry system is tied strongly to the stability of the predator-prey system. However, when the systems are weakly coupled (little biomass is transferred through the quarry, perhaps because it has a much lower utility to the scavenger than the resource), then the resource-scavenger-quarry system will be more robust against changes in the predator-prey system dynamics.

If the predator-prey and resource-scavenger-quarry systems are on two different timescales, then it may be more difficult to ascertain the influence of the first on the latter. As an example, when the predator-prey system evolves on a faster timescale than does the resource-scavenger-quarry system, there may be slow chaotic dynamics which might be mistaken for periodic dynamics if one takes a small sample size of observations. Therefore, the understanding the the interlinked dynamics from these model may be relevant for ascertaining whether such coupled ecological systems are really in sync or if the resource-scavenger-quarry system falls out of sync with the predator-prey systems. This can lead to less regularity in boom-and-bust dynamics, making bust events, which may require external intervention to conserve a particular species, harder to predict.

In Levy et al. (2016), it was found that increasing the amplitude of the death rate of the foxes $\delta(t)$ could push them to extinction. We see that in this case, the population 
of foxes becomes so large, that it drives the lemming population to extinction. This type of dynamics is known as 'boom and bust' dynamics and is indeed observed in nature (see for example Dickman et al. (2010) or Kingsford et al. (1999)). In PPQRS dynamics, we observe similar boom and bust behaviours from non-autonomous contributions to the death rate of the predators, $\beta(t)$. We saw that the addition of non-autonomous seasonal terms can lead to chaos. In Oksanen and Oksanen (1992), the population sizes of Arctic lemmings and subarctic voles were compared. Where the lemming dynamics were chaotic, the vole dynamics were found to be periodic. In Levy et al. (2016), it was suggested that large temperature differences on the Arctic could be responsible for the chaotic dynamics of the lemmings. Other explanations are also possible, such as defensive chemicals produced by plants as response to grazing by the lemmings (Seldal et al. 1994). Finally, in the cases that the autonomous system showed chaotic behaviour already, we found that the addition of non-autonomous terms could, for certain parameter values, resolve this chaos by pushing the resource to extinction or other steady states.

We observed spatial patterning when multiple species were permitted to move throughout a spatial domain via diffusion. As in the purely temporal dynamics, a variety of spatially uniform steady states were possible for such models, yet additional spatially heterogeneous dynamics were apparent up to large times in numerical simulations. These states often correspond to limit cycles in the purely time-dependent model, only with the amplitude of the fluctuations varying as a function of spatial position, we well. It may be possible that some of the structures observed correspond to spatio-temporal chaos.

Ecologically, the spatial dynamics are interesting as fluctuations in populations appear to be less extreme than in the purely time-dependent model. This suggests that making heterogeneous spatial regions available can mitigate boom-and-bust dynamics which might otherwise lead to extinction events. This might be one reason why boom-andbust dynamics associated with the paradox of enrichment are hard to find in real-world examples. As many real-world examples have some degree of spatial heterogeneity, it may be that very extreme fluctuations in populations due to an increase in, say, carrying capacity of the prey are strongly moderated by spatial diffusion processes. The moderating role of spatial diffusion in a PDE system for the predatorprey-subsidy system alone was recently studied in Bassett et al. (2017). In Bassett et al. (2017), the dynamics usually resulted in solutions which, while heterogeneous, had a degree of spatial symmetry. The kinds of irregular heterogeneous patterns we observe here (such as in Figs. 8 and 10) were not found, implying that coupling of the two systems as we do results in more exotic dynamics, and hence, that more variation is possible in real-world systems which are interconnected rather than modelled in isolation. However, this may also be due to the fact that there was only one predator in that model, whereas in our model, the top predator is confined to one region while the secondary predator (the scavenger) has mobility over the entire domain, thereby complicating and possibly destabilizing the model dynamics.

Conversely, these findings for the spatial model may suggest that limiting the spatial region available to a particular population may destabilize the population dynamics. Such results were recently shown on discrete stepping stone domains for the predator-prey-subsidy system in Shen and Van Gorder (2017). In that paper, variations in the underlying network structure or topology were seen to alter the structure of bifurcations from equilibrium values to nonequilibrium dynamics. Spatial structure, particularly heterogeneous structures where certain populations are confined to subsets of the whole environment, can therefore modify stability of predator-prey dynamics. This is an important point for conservation ecology, as destruction of habitat may result in such a stability loss.

\section{Conclusions}

The main difference between our PPQRS model given by Eqs. 2.1-2.5 and the PPS model of (Nevai and Van Gorder 2012) is the addition of an extra limit cycle in the predatorprey system, which can destabilize the PPQRS systems in ways not possible in the PPS system. Because of this limit cycle, we found that the resource-free equilibrium is not stable for any value of $c$ or $k$, whereas in the PPS model of Nevai and Van Gorder (2012), this equilibrium can always be made stable. The existence of a second limit cycle does also result in the occurrence of quasi-periodic and aperiodic solutions, which, again, were not found in the PPS model of Nevai and Van Gorder (2012). In Levy et al. (2016), a PPS model with a non-constant input rate of the form (7.2). This oscillating input rate did interact with the existing limit cycle of the scavengers and resources, resulting in quasi-periodic solutions. However, the simple sinusoidal form of Eq. 7.2 did not generate chaotic solutions, while our autonomous PPQRS model did give us chaos.

We found that adding non-autonomous terms (in order to model seasonality) can fundamentally modify dynamics of the autonomous system, turning equilibria into periodic solutions, and periodic solutions into quasi-periodic and even aperiodic solutions. These results agree with the findings in Levy et al. (2016) for their PPS system. Adding seasonality can both prevent species from extinction and drive species to extinction. Although some of these results could have been found for non-autonomous PP and PPS models as well, we have not found them in the existing 
literature. In the cases where the autonomous system already exhibited chaotic behaviour, we found that the addition of non-autonomous terms could, for certain parameter values, resolve this chaos by pushing the resource to extinction or to other steady states. Our findings show that the predator-prey dynamics under seasonal terms (non-autonomous forcing) can have a large influence on the resource and scavenger populations.

We investigated a spatial PDE model numerically. We were especially interested in the interaction of the scavenger dynamics on the two different domains we introduced: a resource-free and a quarry-free area. In cases where the ODE model showed limit cycle behaviour, the PDE model showed spatially non-homogeneous solutions that were oscillating in time. In situations where increasing the carrying capacity in the ODE model drives the amplitude of the oscillations up, we found that a large carrying capacity in the PDE model results in a very small variation in average population size. That the addition of spatial variation can decrease the average amplitude of the oscillations has been observed before in Petrovskii et al. (2004) for a simple predator-prey system with one limit cycle. We extended these results to the chaotic solutions that we found in our autonomous ODE model, showing that there, too, the addition of spatial variation can smooth out the effects of high carrying capacities. Finally, we found that when the amount of quarry is not high enough, scavengers will not enter the predator-prey-quarry area. This difference with our ODE model is especially important for large domains or slow rates of diffusion, when scavengers are more sensitive to the area in which they will live, as they remain for a larger period of time.

Open Access This article is distributed under the terms of the Creative Commons Attribution 4.0 International License (http://creative commons.org/licenses/by/4.0/), which permits unrestricted use, distribution, and reproduction in any medium, provided you give appropriate credit to the original author(s) and the source, provide a link to the Creative Commons license, and indicate if changes were made.

\section{Appendix A: Local stability for steady states of the autonomous model}

We will consider the feasibility, local and global stability of the equilibrium states of our autonomous model, given by Eqs. 2.1-2.5, where we define a state state as a solution of our model that is constant in time. At a steady-state point $\left(u^{*}, v^{*}, s^{*}, x^{*}, y^{*}\right)$, the equations satisfy

$0=\rho u^{*}\left(1-\frac{u^{*}}{c}\right)-\phi\left(\frac{u^{*} v^{*}}{u^{*}+g}\right)$,

$0=\left(\frac{\zeta \phi u^{*}}{u^{*}+g}\right) v^{*}-\beta v^{*}$, $0=\mu \phi\left(\frac{u^{*} v^{*}}{u^{*}+g}\right)-\gamma s^{*}-\psi\left(\frac{s^{*} y^{*}}{x^{*}+s^{*}+h}\right)$,

$0=r x^{*}\left(1-\frac{x^{*}}{k}\right)-\theta\left(\frac{x^{*} y^{*}}{x^{*}+s^{*}+h}\right)$,

$0=\left(\frac{\epsilon \theta x^{*}+\eta \psi s^{*}}{x^{*}+s^{*}+h}\right) y^{*}-\delta y^{*}$.

The Jacobian of the system is given by

$J_{\left(u^{*}, v^{*}, s^{*}, x^{*}, y^{*}\right)}=\left(\begin{array}{ccccc}J_{11} & J_{12} & 0 & 0 & 0 \\ J_{21} & J_{22} & 0 & 0 & 0 \\ J_{31} & J_{32} & J_{33} & J_{34} & J_{35} \\ 0 & 0 & J_{43} & J_{44} & J_{45} \\ 0 & 0 & J_{53} & J_{54} & J_{55}\end{array}\right)$,

where

$J_{11}=\frac{\phi u^{*} v^{*}}{\left(g+u^{*}\right)^{2}}-\frac{\phi v^{*}}{g+u^{*}}-\rho\left(2 \frac{u^{*}}{c}-1\right)$,

$J_{12}=-\frac{\phi u^{*}}{g+u^{*}}, J_{21}=\frac{\phi v^{*} \zeta}{g+u^{*}}-\frac{\phi u^{*} v^{*} \zeta}{\left(g+u^{*}\right)^{2}}$,

$J_{22}=\frac{\phi u^{*} \zeta}{g+u^{*}}-\beta, J_{31}=\frac{\mu \phi v^{*}}{g+u^{*}}-\frac{\mu \phi u^{*} v^{*}}{\left(g+u^{*}\right)^{2}}, J_{32}=\frac{\mu \phi u^{*}}{g+u^{*}}$,

$J_{33}=\frac{\psi s^{*} y^{*}}{\left(h+s^{*}+x^{*}\right)^{2}}-\frac{\psi y^{*}}{h+s^{*}+x^{*}}-\gamma$,

$J_{34}=\frac{\psi s^{*} y^{*}}{\left(h+s^{*}+x^{*}\right)^{2}}, J_{35}=-\frac{\psi s^{*}}{h+s^{*}+x^{*}}$,

$J_{43}=\frac{\theta x^{*} y^{*}}{\left(h+s^{*}+x^{*}\right)^{2}}$,

$J_{44}=\frac{\theta x^{*} y^{*}}{\left(h+s^{*}+x^{*}\right)^{2}}-\frac{\theta y^{*}}{h+s^{*}+x^{*}}-r\left(2 \frac{x^{*}}{k}-1\right)$,

$J_{45}=-\frac{\theta x^{*}}{h+s^{*}+x^{*}}$,

$J_{53}=\frac{\eta \psi y^{*}}{h+s^{*}+x^{*}}-\frac{y\left(\eta \psi s^{*}+\epsilon \theta x^{*}\right)}{\left(h+s^{*}+x^{*}\right)^{2}}$,

$J_{54}=\frac{\epsilon \theta y^{*}}{h+s^{*}+x^{*}}-\frac{y\left(\eta \psi s^{*}+\epsilon \theta x^{*}\right)}{\left(h+s^{*}+x^{*}\right)^{2}}$,

$J_{55}=\frac{\eta \psi s^{*}+\epsilon \theta x^{*}}{h+s^{*}+x^{*}}-\delta$.

\section{A.1 The Rosenzweig-MacArthur model}

To find the steady states of our model, we first consider only the predator and quarry Eqs. A.1 and (A.2), because those can be decoupled from the system. Note that these two equations form the classic Rosenzweig-MacArthur model. It is well known that the steady states of this model are given by $(0,0),(c, 0)$ and $\left(u^{*}, v^{*}\right)$, where

$$
u^{*}=\frac{g \beta}{\zeta \phi-\beta}, \quad v^{*}=\frac{\rho}{\phi}\left(1-\frac{u^{*}}{c}\right)\left(u^{*}+g\right) \text {. }
$$

We define

$c=\frac{g \beta}{\zeta \phi-\beta}, \quad \tilde{c}=\frac{g(\zeta \phi+\beta)}{\zeta \phi-\beta}$. 
Note that $0<\underset{\sim}{\underset{c}{c}<}<\underset{\sim}{c}+g=\tilde{c}$. The trivial steady state of the Rosenzweig-McArthur model $(0,0)$ is always unstable, and $(c, 0)$ is stable if and only if $c<\underset{\sim}{c}$. Lastly, $\left(u^{*}, v^{*}\right)$ is feasible if and only if $\underset{\sim}{c}<c$. If $\underset{\sim}{c}<c<\tilde{c}, u \rightarrow u^{*}$ and $v \rightarrow$ $v^{*}$, but when $c>\tilde{c},\left(u^{*}, v^{*}\right)$ becomes unstable and turns into a single stable limit cycle, due to a Hopf bifurcation (see, for example, Hofbauer and Sigmund (1998) for a more thorough discussion of the above results).

\section{A.2 The full PPQRS model}

We turn to the full five-dimensional model (2.1)-(2.5). We examined the feasibility and local stability of the equilibrium points, by looking at the sign of the eigenvalues of the Jacobian of the linearised system for all stable points. There exist five possibly stable steady states, which we will call the predator- and scavenger-free $(c, 0,0, k, 0)$, the predatorfree $\left(c, 0,0, x^{*}, y^{*}\right)$, the scavenger-free $\left(u^{*}, v^{*}, \frac{i}{\gamma}, k, 0\right)$, the resource-free $\left(u^{*}, v^{*}, \underline{l}, 0, \frac{\eta}{\delta}\left(i-\gamma s^{*}\right)\right)$ and the positive equilibrium $\left(u^{*}, v^{*}, s^{*}, x^{*}, y^{*}\right)$, respectively. Consider the first steady state of (A.1) and (A.2), where $u=v=0$. In this case, there are neither prey nor predators and consequently, no quarry is created. Then, our model reduces two a standard two-species model with only scavengers and resources. So $u=v=0$ leads to the following three steady states: $(0,0,0,0,0),(0,0,0, k, 0)$ and $\left(0,0,0, x^{*}, y^{*}\right)$. These equilibria are never stable, as the Jacobian $J$ has at least one eigenvalue with positive real part, i.e. $\lambda_{1}=\rho>0$.

At the second steady state of Eqs. A.1 and A.2, there are no predators. Again, no quarry is created and the model reduces to a system of scavengers, resources and unhunted prey. As we saw above, this state can be stable if $c<\underset{\sim}{c}$. We find the three steady states $(c, 0,0,0,0),(c, 0,0, k, 0)$ and $\left(c, 0,0, x^{*}, y^{*}\right)$. We have

$$
J_{(c, 0,0,0,0)}=\left(\begin{array}{ccccc}
-\rho & -\frac{\phi c \zeta}{g+c} & 0 & 0 & 0 \\
0 & \frac{\phi c \zeta}{g+c}-\beta & 0 & 0 & 0 \\
0 & \frac{\mu \phi c}{g+c} & -\gamma & 0 & 0 \\
0 & 0 & 0 & r & 0 \\
0 & 0 & 0 & 0 & -\delta
\end{array}\right),
$$

which is never stable because $r>0$. The next equilibrium point gives

$$
J_{(c, 0,0, k, 0)}\left(\begin{array}{ccccc}
-\rho & -\frac{\phi c \zeta}{g+c} & 0 & 0 & 0 \\
0 & \frac{\phi c \zeta}{g+c}-\beta & 0 & 0 & 0 \\
0 & \frac{\mu \phi c}{g+c} & -\gamma & 0 & 0 \\
0 & 0 & 0 & -r & -\frac{\theta k}{h+s+k} \\
0 & 0 & 0 & 0 & \frac{\epsilon \theta k}{h+s+k}-\delta
\end{array}\right) .
$$

The characteristic polynomial is given by

$$
\begin{aligned}
\chi(\lambda)= & (-\rho-\lambda)\left(\frac{\phi c \zeta}{g+c}-\beta-\lambda\right)(-\gamma-\lambda)(-r-\lambda) \\
& \times\left(\frac{\epsilon \theta k}{h+s+k}-\delta-\lambda\right) .
\end{aligned}
$$

If and only if $c<\underset{\sim}{c}$ and $k<\underset{\sim}{k}=\frac{h \delta}{\epsilon \theta-\delta}$, all eigenvalues are negative and the point is stable. Finally,

$$
J_{\left(c, 0,0, x^{*}, y^{*}\right)}=\left(\begin{array}{ccccc}
J_{11} & J_{12} & 0 & 0 & 0 \\
0 & J_{22} & 0 & 0 & 0 \\
0 & J_{32} & J_{33} & 0 & 0 \\
0 & 0 & J_{43} & J_{44} & J_{45} \\
0 & 0 & J_{53} & J_{54} & J_{55}
\end{array}\right) .
$$

Notice that the lower right $(2 \times 2)$ block reduces to a predator prey relation as the one we considered above. We deduce that this block has a feasible positive equilibrium $\left(x^{*}, y^{*}\right)$ if and only if $k>\underset{\sim}{k}$ and its eigenvalues are negative if and only of $\underset{\sim}{k}<k<\tilde{k}$, where $\underset{\sim}{k}=\frac{h \delta}{\epsilon \theta-\delta}$ and $\tilde{k}=\frac{h(\epsilon \theta+\delta)}{\epsilon \theta-\delta}$. The characteristic polynomial of Eq. A.12 is given by

$$
\begin{aligned}
\chi(\lambda)= & (-\rho-\lambda)\left(\frac{\phi c \zeta}{g+c}-\beta-\lambda\right)\left(-\frac{\psi y^{*}}{h+x^{*}}-\gamma-\lambda\right) \\
& \times \operatorname{det}\left(J_{(4: 5,4: 5)}-\lambda I\right) .
\end{aligned}
$$

So, $\left(c, 0,0, x^{*}, y^{*}\right)$ is stable if and only if $c<\underset{c}{c}$ and $\underset{\sim}{k}<$ $k<\tilde{k}$. When $\underset{\sim}{c}<c<\tilde{c},\left(u^{*}, v^{*}\right)$ is a stable equilibrium of (A.1) and (A.2). Then, Eqs. A.3-A.5 become

$0=\mu \rho \underset{\sim}{c}\left(1-\frac{c}{c}\right)-\gamma s-\psi\left(\frac{s y}{x+s+h}\right)$,

$0=r x\left(1-\frac{x}{k}\right)-\theta\left(\frac{x y}{x+s+h}\right)$,

$0=\left(\frac{\epsilon \theta x+\eta \psi s}{x+s+h}\right) y-\delta y$.

We define $i=\mu \rho \underset{\sim}{c}\left(1-\frac{c}{c}\right)$ and write our system as

$\frac{d s}{d t}=i-\gamma s-\psi\left(\frac{s y}{x+s+h}\right)$,

$\frac{d x}{d t}=r x\left(1-\frac{x}{k}\right)-\theta\left(\frac{x y}{x+s+h}\right)$,

$\frac{d y}{d t}=\left(\frac{\epsilon \theta x+\eta \psi s}{x+s+h}\right) y-\delta y$.

In Nevai and Van Gorder (2012), Nevai and Van Gorder fully analysed above PPS model with constant input rate $i$. In our following analysis, we will make use of their results.

The system (A.17)-(A.19) has the four equilibrium points

$\left(\frac{i}{\gamma}, 0,0\right),\left(\frac{i}{\gamma}, k, 0\right),\left(\underline{l}, 0, \frac{\eta}{\delta}(i-\gamma \underline{l})\right)$ and $\left(s^{*}, x^{*}, y^{*}\right)$, 
where

$\underline{l}=\frac{h \delta}{\eta \psi-\delta}$.

The first two equilibria are always feasible, the prey-free equilibrium is feasible if and only if $i>\gamma \underline{l}$ and the positive equilibrium is feasible if and only if $i<i^{*}$ and, when $k<\underset{\sim}{k}$, $i_{*}(k)<i<i^{*}$. Here, $i_{*}(k)$ and $i^{*}$ are defined as

$i_{*}(k)=\left(1-\frac{k}{\underset{\sim}{k}}\right) \underline{l} \gamma, \quad i^{*}=\left(\gamma+\frac{r \psi}{\theta}\right) l$.

We turn again to the full system and examine the local stability of the steady states $\left(u^{*}, v^{*} \frac{i}{\gamma}, 0,0\right),\left(u^{*}, v^{*} \frac{i}{\gamma}\right.$, $k, 0),\left(u^{*}, v^{*}, \underline{l}, 0, \frac{\eta}{\delta}(i-\gamma \underline{l})\right)$, and $\left(u^{*}, v^{*}, s^{*}, x^{*}, y^{*}\right)$.

For $\left(u^{*}, v^{*}, \frac{i}{\gamma}, 0,0\right)$, the Jacobian takes the form

$J_{\left(u^{*}, v^{*}, \frac{i}{\gamma}, 0,0\right)}=\left(\begin{array}{ccccc}J_{11} & J_{12} & 0 & 0 & 0 \\ J_{21} & J_{22} & 0 & 0 & 0 \\ J_{31} & J_{32} & J_{33} & 0 & J_{35} \\ 0 & 0 & 0 & J_{44} & 0 \\ 0 & 0 & 0 & 0 & J_{55}\end{array}\right)$.

The characteristic polynomial is of the form

$$
\begin{aligned}
\chi(\lambda)= & \operatorname{det}\left(J_{(1: 2,1: 2)}-\lambda I\right)(-\gamma-\lambda)(r-\lambda) \\
& \times\left(\frac{\eta \psi \frac{i}{\gamma}}{h+\frac{i}{\gamma}}-\delta-\lambda\right) .
\end{aligned}
$$

Because $r>0$, this equilibrium point is always unstable.

For $\left(u^{*}, v^{*}, \frac{i}{\gamma}, k, 0\right)$, the Jacobian takes the form

$$
J_{\left(u^{*}, v^{*}, \frac{i}{\gamma}, k, 0\right)}=\left(\begin{array}{ccccc}
J_{11} & J_{12} & 0 & 0 & 0 \\
J_{21} & J_{22} & 0 & 0 & 0 \\
J_{31} & J_{32} & J_{33} & 0 & J_{35} \\
0 & 0 & 0 & J_{44} & J_{45} \\
0 & 0 & 0 & 0 & J_{55}
\end{array}\right) .
$$

The characteristic polynomial is of the form

$$
\begin{array}{r}
\chi(\lambda)=\operatorname{det}\left(J_{(1: 2,1: 2)}-\lambda I\right)(-\gamma-\lambda)(-r-\lambda) \\
\times\left(\frac{\eta \psi \frac{i}{\gamma}+\epsilon \theta k}{h+\frac{i}{\gamma}+k}-\delta-\lambda\right) .
\end{array}
$$

It follows that the equilibrium point is stable if and only if $\underset{\sim}{c}<c<\tilde{c}$ and

$$
\frac{\eta \psi \frac{i}{\gamma}+\epsilon \theta k}{h+\frac{i}{\gamma}+k}<\delta .
$$

The second condition is equivalent to $k<\underset{\sim}{k}$ and $i<i_{*}(k)$.
For $\left(u^{*}, v^{*}, \underline{l}, 0, \frac{\eta}{\delta}(i-\gamma \underline{l})\right)$, the Jacobian takes the form

$J_{\left(u^{*}, v^{*}, \underline{l}, 0, \frac{\eta}{\delta}(i-\gamma \underline{l})\right)}=\left(\begin{array}{ccccc}J_{11} & J_{12} & 0 & 0 & 0 \\ J_{21} & J_{22} & 0 & 0 & 0 \\ J_{31} & J_{32} & J_{33} & J_{34} & J_{35} \\ 0 & 0 & 0 & J_{44} & 0 \\ 0 & 0 & J_{53} & J_{54} & 0\end{array}\right)$.

The characteristic polynomial is of the form

$$
\begin{aligned}
\chi(\lambda)= & \left(J_{55}-\lambda\right)\left(J_{44}-\lambda\right)\left(J_{33}-\lambda\right) \operatorname{det}\left(J_{(1: 2,1: 2)}-\lambda I\right) \\
& -J_{35} J_{53} J_{44} \operatorname{det}\left(J_{(1: 2,1: 2)}-\lambda I\right) \\
= & \left(-J_{35} J_{53}+(-\lambda)\left(J_{33}-\lambda\right)\right)\left(J_{44}-\lambda\right) \operatorname{det} \\
& \times\left(J_{(1: 2,1: 2)}-\lambda I\right) .
\end{aligned}
$$

It follows that the equilibrium point is stable if and only if $\underset{\sim}{c}<c<\tilde{c}, J_{44}<0$ and the equation

$\lambda^{2}-J_{33} \lambda-J_{35} J_{53}=0$,

has two roots with negative real part. The condition $J_{44}<0$ is satisfied if and only if $i=\mu \rho \underset{\sim}{c}\left(1-\frac{\underset{c}{c}}{c}\right)>i^{*}$ (see Nevai and Van Gorder (2012, p. 897)). For the negative roots, we need $J_{33}<0$ and $J_{35} J_{53}<0$. Rewriting

$J_{33}=-\left(\frac{\psi h \frac{\eta}{\delta}(i-\gamma \underline{l})}{(h+\underline{l})^{2}}\right)-\gamma$

and

$J_{35} J_{53}=-\left(\frac{\psi^{2} \underline{l} h \eta \frac{\eta}{\delta}(i-\gamma \underline{l})}{(h+\underline{l})^{3}}\right)$,

we see that $J_{33}<0$ and $J_{35} J_{53}<0$ is always satisfied.

We conclude that $\left(u^{*}, v^{*}, \underline{l}, 0, \frac{\eta}{\delta}(i-\gamma \underline{l})\right)$ is stable if and only if $\underset{\sim}{c}<c<\tilde{c}$ and $i=\mu \rho \underset{\sim}{c}\left(1-\frac{\underset{c}{c}}{c}\right)>i^{*}$.

We finally turn to the steady state $\left(u^{*}, v^{*}, x^{*}, y^{*}, s^{*}\right)$. The corresponding Jacobian takes the form

$J=\left(\begin{array}{ccccc}J_{11} & J_{12} & 0 & 0 & 0 \\ J_{21} & 0 & 0 & 0 & 0 \\ J_{31} & J_{32} & J_{33} & J_{34} & J_{35} \\ 0 & 0 & J_{43} & J_{44} & J_{45} \\ 0 & 0 & J_{53} & J_{54} & 0\end{array}\right)$

We still assume $\underset{\sim}{c}<c<\tilde{c}$, so that the number of prey and predators is stable. In Nevai and Van Gorder (2012), Nevai and Van Gorder found $\left(s^{*}, x^{*}, y^{*}\right)$ to be feasible in three different cases:

(i) $k<\underset{\sim}{k}$ and $i_{*}(k)<i<i^{*}$,

(ii) $\underset{\sim}{k}<\hat{k}<\tilde{k}$ and $i<i^{*}$,

(iii) $k>\tilde{k}$ and $i<i^{*}$.

Therefore, we will determine the local stability in these three different cases. For this, we will look at the signs of the different elements of Eq. A.30. 
Rewriting $J_{31}$ as $J_{31}=\left(\frac{\mu \phi u^{*} v^{*} g}{\left(g+u^{*}\right)^{2}}\right)$, we see that $J_{31}$ is always positive. Further, we see that $J_{32}, J_{34}$ and $J_{43}$ are always positive and $J_{45}$ and $J_{35}$ are always negative. Rewriting $J_{33}$ as $J_{33}=-\left(\frac{h+x^{*}}{\left(h+s^{*}+x^{*}\right)^{2}}\right)-\gamma$, we see that $J_{33}$ is always negative as well. We rewrite $J_{53}$ and $J_{54}$ as

$$
\begin{aligned}
J_{53} & =\frac{y^{*}\left(\eta \psi x^{*}+\eta \psi h-\epsilon \theta x^{*}\right)}{\left(h+s^{*}+x^{*}\right)^{2}} \\
& =(\eta \psi-\delta) \frac{y^{*}}{\left(h+s^{*}+x^{*}\right)}>0,
\end{aligned}
$$

and

$$
\begin{aligned}
J_{54} & =\frac{y^{*}\left(\epsilon \theta s^{*}+\epsilon \theta h-\eta \psi s^{*}\right)}{\left(h+s^{*}+x^{*}\right)^{2}} \\
& =(\epsilon \theta-\delta) \frac{y^{*}}{\left(h+s^{*}+x^{*}\right)}>0,
\end{aligned}
$$

since $\eta \psi, \epsilon \theta>\delta$. Finally, following (Nevai and Van Gorder 2012), we can reduce $J_{44}$ to

$$
J_{44}=\frac{r x^{*}}{x^{*}+s^{*}+h}\left(1-\frac{2 x^{*}+s^{*}+h}{k}\right) .
$$

It follows that $J_{44}<0$ if and only if $k<\bar{k}=2 x^{*}+s^{*}+h$. Consequently, the Jacobian (A.30) has sign pattern:

$\operatorname{sgn}(J)=\left(\begin{array}{ccccc}- & - & 0 & 0 & 0 \\ + & 0 & 0 & 0 & 0 \\ + & + & - & + & - \\ 0 & 0 & + & \operatorname{sgn}(k-\bar{k}) & - \\ 0 & 0 & + & + & 0\end{array}\right)$.

The characteristic polynomial is given by

$$
\begin{aligned}
\chi(\lambda)= & \left(J_{11}-\lambda\right)(-\lambda) \operatorname{det}\left(J_{(3: 5,3: 5)}-\lambda I\right) \\
& -J_{12} J_{21} \operatorname{det}\left(J_{(3: 5,3: 5)}-\lambda I\right) \\
= & \left(\lambda^{2}-\lambda J_{11}-J_{12} J_{21}\right) \operatorname{det}\left(J_{(3: 5,3: 5)}-\lambda I\right) .
\end{aligned}
$$

Now, because $J_{21} J_{12}<0$ and $J_{11}<0$, the positive equilibrium is stable if and only if $J_{(3: 5,3: 5)}$ has eigenvalues with negative real part. We can write the characteristic polynomial of $J_{(3: 5,3: 5)}$ as

$a_{0} \lambda^{3}+a_{1} \lambda^{2}+a_{2} \lambda+a_{3}$,

with $a_{0}=1, a_{1}=-\operatorname{tr}\left(J_{(3: 5,3: 5)}\right), a_{2}=J_{44} J_{33}-\left(J_{34} J_{43}+\right.$ $\left.J_{35} J_{53}+J_{45} J_{54}\right)$ and $a_{3}=-\operatorname{det}\left(J_{(3: 5,3: 5)}\right)$. We use the Routh-Hurwitz criterion: in order for the roots of a polynomial of the form (A.36) with real coefficients and $a_{0} \neq 0$ to have all negative real parts, it is necessary and sufficient that $a_{0} a_{1}>0$ and $a_{1} a_{2}>a_{0} a_{3}$ (Gantmacher 1959, p. 231). When $k<\tilde{k}$, we have $\operatorname{tr}\left(J_{(3: 5,3: 5)}\right)<0$ and $\operatorname{det}\left(J_{(3: 5,3: 5)}\right)<$ 0 . It follows that $J$ is stable if and only if $a_{1} a_{2}>a_{3}$. Unfortunately, it is complicated to determine when this inequality holds, based on parameter values alone. When $k>\tilde{k}$, it becomes even more difficult to determine the roots of the polynomial (Nevai and Van Gorder 2012, p. 901). Therefore, following (Nevai and Van Gorder 2012), we have numerically investigated the stability of the system.

\section{Appendix B: Lyapunov exponents}

This appendix consists of an introduction to Lyapunov exponents, based on the theoretical discussion in Nayfeh and Balachandran (1995, p. 525-529). Consider an $n$ dimensional autonomous system of differential equations:

$\frac{d \mathbf{x}}{d t}=\mathbf{f}(\mathbf{x})$

with a trajectory $\mathbf{X}(t)$ and a small perturbation $\delta(t)$ of this trajectory. We linearise around the perturbation to obtain

$\frac{d \delta}{d t}=A(t) \delta(t)$

with $A(t)$ the Jacobian matrix, which is time-dependent. The solution of this problem is given by

$\delta(t)=\Phi(t) \delta(0)$

where $\Phi(t)$ is the fundamental matrix of the problem. The rate of exponential grow or decay in the direction of $\delta(0)$ is given by

$\bar{\lambda}_{i}=\lim _{t \rightarrow \infty} \frac{1}{t} \ln \left(\frac{\|\delta(t)\|}{\|\delta(0)\|}\right)$.

We call $\bar{\lambda}_{i}$ a Lyapunov exponent. We can find $n$ linearly independent perturbation vectors $\delta, i=1, \ldots n$, corresponding to the $n$ Lyapunov exponents of our system. The basis $\delta, i=1, \ldots n$ is called a normal basis if

$\sum_{i=1}^{n} \bar{\lambda}_{i}\left(\delta_{i}\right) \leq \sum_{i=1}^{n} \bar{\lambda}_{i}\left(\tilde{\delta}_{i}\right)$

for all other bases $\tilde{\delta}, i=1, \ldots n$. Normal bases are not unique. Just like the eigenvalues of a matrix that are invariant under a change of basis, the Lyapunov exponents of a system depend on $\Phi(t)$ and not on the choice of normal basis. For a fixed point of the system, the Lyapunov exponents are given by

$\bar{\lambda}_{i}=\lim _{t \rightarrow \infty} \frac{1}{t} \operatorname{Re}\left(\lambda_{i} t\right)$,

with $\lambda_{i}$ an eigenvalue of the Jacobian matrix $A$ of the linearised system around the fixed point. When all the real parts of the eigenvalues are negative, all Lyapunov exponents are negative as well and we have a stable fixed point. Periodic cycles always have one Lyapunov exponent equal to zero, corresponding to a perturbation $\delta(t)$ along a tangent 
to the cycle $\mathbf{X}(t)$. When we have a stable limit cycle, the other Lyapunov exponents must be negative, corresponding to perturbations in directions normal to the trajectory. An $n$-period quasi-periodic solution has $n$ zero Lyapunov exponents, corresponding to the $n$ tangential directions on the torus. Finally, one or more positive Lyapunov exponents are a sign of chaotic behaviour. A positive Lyapunov exponent corresponds to a perturbation direction in which a trajectory that is separated from the original trajectory will diverge.

\section{References}

Abrams P, Holt R, Roth J (1998) Apparent competition or apparent mutualism? Shared predation when populations cycle. Ecology 79(1):201-212

Alonso D, Bartumeus F, Catalan J (2002) Mutual interference between predators can give rise to Turing spacial patterns. Ecology 83(1):28-34

Bassett A, Krause AL, Van Gorder RA (2017) Continuous dispersal in a model of predator-prey-subsidy population dynamics. Ecol Model 354:115-122

Berg M, Hellenthal B (1992) The role of Chironomidae in energy flow of a lotic ecosystem. Neth J Aquat Ecol 26(2):471-476

Berryman A (1992) The origins and evolution of predator-prey theory. Ecology 73(5):1530-1535

Cheng KS (1981) Uniqueness of a limit cycle for a predator-prey system. SIAM J Math Anal 12:541

DeAngelis DL (1980) Energy flow, nutrient cycling, and ecosystem resilience. Ecology 61(4):764-771

DeAngelis DL, Bartell SM, Brenkert AL (1989) Effects of nutrient recycling and food-chain length on resilience. Am Nat 134:778805

Dickman CR, Greenville AC, Beh CL, Tamayo BM, Wardle G (2010) Social organization and movements of desert rodents during population "booms" and "busts" in central Australia. J Mammal 91(4):798-810

Edelstein-Keshet L (2005) Mathematical models in biology (Classics in applied mathematics ; 46). Philadelphia: Society for Industrial and Applied Mathematics

Fuller WA, Martell AM, Smith RFC, Speller SW (1975) High-Arctic lemmings, Dicrostonyx groenlandicus. II. Demography. Can J Zool 53(6):867-878

Gantmacher F (1959) Applications of the theory of matrices. Interscience, New York

Gasull A, Kooij R, Torregrosa J (1997) Limit cycles in the HollingTanner model. Publicacions Matemàtiques 41:149-167

Gause G, Smaragdova N, Witt A (1936) Further studies of interaction between predators and prey. J Anim Ecol 5(1):1-18

Govorukhin V (2004) Lyapunov exponents toolbox. https://www.math works.com/matlabcentral/fileexchange/4628-calculation-lyapunovexponents-for-ode. MATLAB Central File Exchange, Retrieved 07-07-2016

Hofbauer J, Sigmund K (1998) Evolutionary games and population dynamics. Cambridge University Press, Cambridge

Holling CS (1959) The components of predation as revealed by a study of small-mammal predation of the European pine sawfly. Can Entomol 91(5):293-320

Holling CS (1965) The functional response of predators to prey density and its role in mimicry and population regulation. Memoirs of the Entomological Society of Canada 97(S45):5-60

Holt R (1977) Predation, apparent competition, and the structure of prey communities. Theor Popul Biol 12(2):197-229
Huxel G, McCann K (1998) Food web stability: the influence of trophic flows across habitats. Am Nat 152(3):460-469

Huxel GR, McCann K, Polis GA (2002) Effects of partitioning allochthonous and autochthonous resources on food web stability. Ecol Res 17(4):419-432

Jones PD, New M, Parker DE, Martin S, Rigor IG (1999) Surface air temperature and its changes over the past 150 years. Rev Geophys 37(2):173-199

Kingsford RT, Curtin AL, Porter J (1999) Water flows on Cooper Creek in arid Australia determine 'boom' and 'bust' periods for waterbirds. Biol Conserv 88(2):231-248

Kuang Y, Freedman HI (1988) Uniqueness of limit cycles in Gausetype models of predator-prey systems. Math Biosci 88(1):67-84

Leslie PH (1948) Some further notes on the use of matrices in population mathematics. Biometrika 35(3/4):213-245

Levy D, Harrington HA, Van Gorder RA (2016) Role of seasonality on predator-prey-subsidy population dynamics. J Theor Biol 396:163-181

Lotka A (1925) Elements of physical biology. Williams Wilkins, Baltimore

May R (2001) Stability and complexity in model ecosystems (Princeton landmarks in biology). Princeton University Press, Oxford

Medvinsky A, Petrovskii S, Tikhonova I, Malchow H, Li B (2002) Spatiotemporal Complexity of plankton and fish dynamics. SIAM Rev 44(3):311-370

Murray J (2002) Mathematical biology (3rd ed., Interdisciplinary applied mathematics ; v. 17). Springer-Verlag, New York

Nayfeh A (1973) Perturbation methods. Wiley-Interscience, New York

Nayfeh A, Balachandran B (1990) Motion near a Hopf bifurcation of a three-dimensional system. Mech Res Commun 17(4):191198

Nayfeh A, Balachandran B (1995) Applied nonlinear dynamics : analytical, computational, and experimental methods (Wiley series in nonlinear science). Wiley, New York

Nevai AL, Van Gorder RA (2012) Effect of resource subsidies on predator-prey population dynamics: a mathematical model. J Biol Dyn 6(2):891-922

Oksanen L, Oksanen T (1992) Long-term microtine dynamics in north Fennoscandian tundra: the vole cycle and the lemming chaos. Ecography 15(2):226-236

Pang PY, Wang M (2004) Strategy and stationary pattern in a threespecies predator-prey model. J Differ Equ 200(2):245-273

Petrovskii S, Li BL, Malchow H (2004) Transition to spatiotemporal chaos can resolve the paradox of enrichment. Ecol Complex $1(1): 37-47$

Pielou E (1969) An introduction to mathematical ecology. WileyInterscience, New York

Real LA (1977) The kinetics of functional response. Am Nat 111:289_ 300

Rinaldi S, Muratori S, Kuznetsov Y (1993) Multiple attractors, catastrophes and chaos in seasonally perturbed predator-prey communities. Bull Math Biol 55(1):15-35

Rose MD, Polis GA (1998) The distribution and abundance of coyotes: the effects of allochthonous food subsidies from the sea. Ecology 79(3):998-1007

Rosenzweig M, MacArthur R (1963) Graphical representation and stability conditions of predator-prey interactions. Am Nat 97(895):209-223

Rosenzweig ML (1971) Paradox of enrichment: destabilization of exploitation ecosystems in ecological time. Science 171(3969): $385-387$

Roth J (2002) Temporal variability in Arctic fox diet as reflected in stable-carbon isotopes; the importance of sea ice. Oecologia 133(1):70-77

Roth J (2003) Variability in marine resources affects Arctic fox population dynamics. J Anim Ecol 72(4):668-676 
Seldal T, Andersen KJ, Hoegstedt G (1994) Grazing-induced proteinase inhibitors: a possible cause for lemming population cycles. Oikos 70(1):3-11

Shen L, Van Gorder RA (2017) Predator-prey-subsidy population dynamics on stepping-stone domains. J Theor Biol 420:241-258

Solomon M (1949) The natural control of animal populations. J Anim Ecol 18(1):1-35

Strogatz S (2014) Nonlinear dynamics and chaos : with applications to physics, biology, chemistry, and engineering (2nd ed.)

Teschl G (2012) Ordinary differential equations and dynamical systems, vol 140. Providence, RI: American Mathematical Society

Turchin P, Hanski I (1997) An empirically based model for latitudinal gradient in vole population dynamics. Am Nat 149(5):842-874

Vandermeer J (2006) Oscillating populations and biodiversity maintenance. BioScience 56(12):967-975
Vannote RL, Minshall GW, Cummins KW, Sedell JR, Cushing CE (1980) The river continuum concept. Can J Fish Aquat Sci 37(1):130-137

Verhulst PF (1845) Recherches mathématiques sur la loi d'accroissement de la population. Nouveaux Mémoires de l'Académie Royale des Sciences et Belles-Lettres de Bruxelles 18:14-54

Vitousek P (1982) Nutrient cycling and nutrient use efficiency. Am Nat 119(4):553-572

Volterra V (1926) Fluctuations in the abundance of a species considered mathematically. Nature 118(2972):558-560

Wolf A, Swift JB, Swinney HL, Vastano JA (1985) Determining Lyapunov exponents from a time series. Physica D 16(3):285-317

Wrigley RE, Hatch DRM (1976) Arctic fox migrations in Manitoba. Arctic 29(3):147-158 